\title{
Antiretroviral drugs induce oxidative stress and neuronal damage in the central nervous system
}

\author{
Cagla Akay • Michael Cooper • Akinleye Odeleye • Brigid K. Jensen • Michael G. White • \\ Fair Vassoler • Patrick J. Gannon • Joseph Mankowski • Jamie L. Dorsey • Alison M. Buch • \\ Stephanie A. Cross • Denise R. Cook • Michelle-Marie Peña • Emily S. Andersen • \\ Melpo Christofidou-Solomidou • Kathryn A. Lindl • M. Christine Zink • Janice Clements • \\ R. Christopher Pierce • Dennis L. Kolson • Kelly L. Jordan-Sciutto
}

Received: 24 October 2013 /Revised: 10 December 2013 / Accepted: 13 December 2013 /Published online: 14 January 2014

(C) The Author(s) 2014. This article is published with open access at Springerlink.com

\begin{abstract}
HIV-associated neurocognitive disorder (HAND), characterized by a wide spectrum of behavioral, cognitive, and motor dysfunctions, continues to affect approximately $50 \%$ of $\mathrm{HIV}(+)$ patients despite the success of combination antiretroviral drug therapy (cART) in the periphery. Of note, potential toxicity of antiretroviral drugs in the central nervous system (CNS) remains remarkably underexplored and may contribute to the persistence of HAND in the cART era. Previous studies have shown antiretrovirals (ARVs) to be neurotoxic in the peripheral nervous system in vivo and in peripheral neurons in vitro. Alterations in lipid and protein metabolism, mitochondrial damage, and oxidative stress all play a role in peripheral ARV neurotoxicity. We hypothesized that ARVs also induce cellular stresses in the CNS, ultimately leading to
\end{abstract}

C. Akay $\cdot$ M. Cooper $\cdot$ A. Odeleye $\cdot$ B. K. Jensen $\cdot$ M. G. White $\cdot$ P. J. Gannon • A. M. Buch • M.-M. Peña $\cdot$ K. A. Lindl •

K. L. Jordan-Sciutto $(\bowtie)$

Department of Pathology, School of Dental Medicine, University of Pennsylvania, 240 S. 40th St, Rm 312 Levy Bldg, Philadelphia,

PA 19104-6030, USA

e-mail: jordank@upenn.edu

F. Vassoler $\cdot$ R. C. Pierce

Department of Psychiatry, The Perelman School of Medicine,

University of Pennsylvania, Philadelphia, PA, USA

J. Mankowski $\cdot$ J. L. Dorsey $\cdot$ M. C. Zink $\cdot$ J. Clements Department of Molecular and Comparative Pathobiology, Johns Hopkins University School of Medicine, Baltimore, MD, USA

S. A. Cross $\cdot$ D. R. Cook $\cdot$ D. L. Kolson

Department of Neurology, The Perelman School of Medicine,

University of Pennsylvania, Philadelphia, PA, USA

E. S. Andersen • M. Christofidou-Solomidou Department of Medicine, The Perelman School of Medicine, University of Pennsylvania, Philadelphia, PA, USA neuronal damage and contributing to the changing clinical and pathological picture seen in HIV-positive patients in the cART era. In this report, we show that ARVs are neurotoxic in the CNS in both pigtail macaques and rats in vivo. Furthermore, in vitro, ARVs lead to accumulation of reactive oxygen species (ROS), and ultimately induction of neuronal damage and death. Whereas ARVs alone caused some activation of the endogenous antioxidant response in vitro, augmentation of this response by a fumaric acid ester, monomethyl fumarate (MMF), blocked ARV-induced ROS generation, and neuronal damage/death. These findings implicate oxidative stress as a contributor to the underlying mechanisms of ARV-induced neurotoxicity and will provide an access point for adjunctive therapies to complement ARV therapy and reduce neurotoxicity in this patient population.

Keywords Antiretroviral · Fumaric acid ester · HIV . HIV-associated neurocognitive disorder · Macaque · Oxidative Stress $\cdot$ Reactive oxygen species $\cdot$ SIV

\section{Introduction}

Despite introduction of combination antiretroviral therapy (cART), HIV-associated neurocognitive disorder (HAND) continues to affect approximately $50 \%$ of $\mathrm{HIV}(+)$ patients (Dore et al. 1999; Heaton et al. 2010). Furthermore, in the cART era, the underlying neuropathology has shifted from overt subcortical involvement to a more insidious cortical damage (Gannon et al. 2011). Various factors, such as poor adherence to drug regimen, emergence of resistant virus species, and residual viral DNA in the central nervous system (CNS), may contribute to these changes (Gannon et al. 2011). However, another likely contributor to HAND in the cART era 
is the virtually unstudied potential for antiretroviral (ARV)related toxicity in the CNS. cART has decreased HIV-related morbidity and mortality by limiting T cell loss and controlling opportunistic infections. However, cART regimens are associated with potentially serious side effects, including dyslipidemia, lipohypertrophy, and increased risk of atherosclerosis (Vidal et al. 2010). Additionally, cART-associated toxicity in the peripheral nervous system is well documented, and it is likely that cART would trigger similar responses in the CNS. Pharmacokinetic studies suggest limited ARV penetrance into the CNS and indicate low cerebrospinal fluid (CSF) and parenchymal drug concentrations (Yilmaz et al. 2004; Yilmaz et al. 2009). However, direct blood-brain barrier (BBB) compromise by viral proteins and neuroinflammation and indirect BBB impairment due to concomitant factors, such as coexisting infections, can lead to increased CSF and parenchymal drug concentrations. Thus, the impact of ARVs in the CNS of $\mathrm{HIV}(+)$ patients is clinically relevant and must be examined.

Initial cART usually includes two nucleoside/nucleotide reverse-transcriptase inhibitors (NRTIs) in combination with a non-nucleoside reverse-transcriptase inhibitor (nNRTI) or with a protease inhibitor (PI) boosted with a low dose of a second PI, Ritonavir. NRTIs and nNRTIs bind to the HIV reversetranscriptase enzyme and inhibit proviral DNA synthesis; PIs inhibit viral proteases needed for virus maturation and assembly. Despite some crossover, certain ARV classes are more highly associated with particular side effects and toxicities than are other classes. PIs alter lipid metabolism and induce the endoplasmic reticulum stress response in macrophages, linking PIs to increased risk of atherosclerosis (Touzet and Philips 2010). NRTIs inhibit DNA polymerase $\gamma$ and lead to decreased mitochondrial DNA, loss of mitochondrial membrane potential, and oxidative phosphorylation, consequently precipitating oxidative stress (Nolan and Mallal 2004). Previous studies exploring possible side effects of ARVs in the CNS are scarce and mostly involve cell lines (Cui et al. 1997). Given the mutations and aberrations in immortalized cell lines, these studies may not reflect the ARV toxicity potentially occurring in biological settings. Peripheral dorsal root ganglia neurons are the only primary cell type of neural lineage previously studied for ARV toxicity (Werth et al. 1994). In this report, we examined effects of ARVs in primary CNS neurons both in vivo and in vitro. Our findings suggest that cART induces oxidative stress and neurotoxicity in the CNS, and that the patients on long-term cART regimens would benefit from adjuvant therapies that include antioxidant strategies to overcome deleterious effects of cART in the CNS.

\section{Materials and methods}

Chemicals and reagents Chemicals and reagents comprise the following: (1) AIDS Research and Reference Reagent
Program, Division of AIDS, NIAID, NIH, antiretroviral reagents; (2) Abcam (Cambridge, MA), mouse monoclonal $\mathrm{NAD}(\mathrm{P}) \mathrm{H} /$ quinone oxidoreductase-1 (NQO-1) antibody (A180) and mouse monoclonal synaptophysin antibody (SY38); (3) BioRad (Hercules, CA), Biosafe Coomassie stain, immunoblot polyvinylidene fluoride (PVDF) membrane and prestained broad range molecular weight ladder; (4) Cell Signaling Technology (Danvers, MA), rabbit polyclonal antibody raised against cleaved caspase-3; (5) Citifluor, Ltd. (London, UK), citifluor AF1. Covance (Princeton, NJ), mouse monoclonal microtubule-associated protein 2 (MAP2) antibody (SMI-52); (6) Dako (Carpinteria, CA), rabbit polyclonal glial fibrillary acidic protein (GFAP) antibody (Z0334); (7) Enzo Life Sciences (Farmingdale, NY), rabbit polyclonal antibody to heme-oxygenase-1 (HO-1); (8) Frontier Scientific (Logan, UT), Sn(IV) mesophorphyrin IX dichloride (SnMP); (9) Jackson ImmunoResearch Labs (West Grove, PA), fluorescein isothiocyanate-conjugated goat anti-mouse $\mathrm{IgG}$ and $\mathrm{Cy} 3-$ conjugated goat anti-rabbit IgG secondary antibodies; (10) Invitrogen (Carlsbad, CA), Dulbecco's modified Eagle's medium (DMEM), tetramethyl rhodamine methyl ester, goat anti-mouse beta-lactamase TEM-1 conjugate, fluorocillin green substrate, dihydroethidium (DHE), 4',6-diamidino-2-phenylindole (DAPI), neurobasal media, and B27 supplement; (11) New England Biolabs (Ipswich, MA), tyramide amplification system; (12) Peptide International (Louisville, KY), poly-L-lysine; (13) Sigma Aldrich (St. Louis, MO), carbonyl cyanide $m$-chlorophenyl hydrazone, cytosine arabinoside (Ara-C), fetal bovine serum (FBS), monomethylfumarate (MMF), oligomycin, propidium iodide, and PI cocktail; (14) ScyTek Labs (Logan, UT), normal antibody diluent; (15) Thermo Scientific (Waltham, MA), goat anti-rabbit horse radish peroxidase (HRP) antibody and goat antimouse HRP antibody, SuperSignal West Dura extended duration substrate; and (16) Tocris Bioscience (Ellisville, MO), thapsigargin. The antibody against calpain-cleaved spectrin was a generous gift from Dr. Robert Siman (The Perelman School of Medicine, University of Pennsylvania, Philadelphia, PA).

Primary cortical neuroglial cultures Primary rat cortical neuroglial, pure neuronal, and pure astrocytic cultures were isolated from embryonic day 17 Sprague Dawley rat pups, with modifications of protocols previously described (Wilcox et al. 1994). Briefly, the cortical cell suspensions isolated from rat pups following standard protocols were plated on poly-Llysine-coated T25 tissue culture flasks $\left(2 \times 10^{6}\right.$ cells/flask $)$, 96-well tissue culture plates $\left(0.5 \times 10^{5}\right.$ cells/well $)$, or glass coverslips $\left(0.25 \times 10^{6}\right.$ cells/well). These cultures were maintained in neurobasal media with $\mathrm{B} 27$ supplement at $37{ }^{\circ} \mathrm{C}$ with $5 \% \mathrm{CO}_{2}$ for the generation of neuroglial cultures, as described previously (Wang et al. 2010; Akay et al. 2011b; White et al. 2011). Half of the media was replaced with fresh media every 7 days and the experiments were performed at 21 days in vitro (DIV), at which time the cultures contain approximately 85 
$90 \%$ neurons and $10-15 \%$ astrocytes/glia. For the preparation of pure neuronal cultures, cultures were treated with $10 \mu \mathrm{M}$ Ara- $\mathrm{C} 48 \mathrm{~h}$ after plating and maintained in neurobasal media with B27 supplement at $37{ }^{\circ} \mathrm{C}$ with $5 \% \mathrm{CO}_{2}$; at 21 DIV, the age at which the experiments were conducted, no astrocytes are detectable by staining for GFAP. Pure astrocytic cultures were prepared by initially plating the cortical cell suspensions in $10 \%$ FBS in DMEM and maintaining at $37^{\circ} \mathrm{C}$ with $5 \% \mathrm{CO}_{2}$ for $7-12$ days, after which time astrocytes constitute more than $90 \%$ of the cultures. These pure astrocytic cultures are then replated in T25 flask or poly-L-lysinecoated glass coverslips in DMEM/10 \% FBS and the experiments are conducted $3-5$ days after replating.

SIV/pigtail macaque model of HIV CNS disease Juvenile pigtailed macaques (Macaca nemestrina) were inoculated with SIV/DeltaB670 and SIV/17E-Fr, as described previously (Zink et al. 1999). Beginning 12 days after inoculation, animals were treated daily with a four ARV drug combination (cART) until necropsy (range, days 161-175). The treatment consisted of the NRTI tenofovir (Gilead) at $30 \mathrm{mg} / \mathrm{kg}$ subcutaneously every day; the PIs saquinavir (Roche) and atazanavir (Bristol- Myers Squibb) at 205 and $270 \mathrm{mg} / \mathrm{kg}$ orally twice a day, respectively; and the integrase inhibitor L-870812 (Merck)(Hazuda et al. 2004) at a dose of $10 \mathrm{mg} / \mathrm{kg}$ orally twice a day(Zink et al. 2010). The tenofovir dose was determined on the basis of previous studies(Tsai et al. 1997), whereas atazanavir and saquinavir doses were determined by pharmacokinetic experiments conducted in pig-tailed macaques, and reflected those that resulted in the same area under the curve as detected in humans treated with atazanavir and saquinavir (Zink et al. 2010). The dose of the integrase inhibitor was based on a previous study conducted in rhesus macaques(Hazuda et al. 2004).

Rodent model of antiretroviral-induced neurotoxicity All surgical procedures were performed with the approval of the Institutional Animal Care and Use Committee. Adult male Sprague Dawley rats were catheterized via jugular vein, as described previously (Thrivikraman et al. 2002). The drug cocktail composed of AZT $\left(100 \mathrm{mg} \mathrm{kg}^{-1} \mathrm{day}^{-1}\right)$, ritonavir (20 mg kg ${ }^{-1}$ day $^{-1}$ ), and saquinavir $\left(25 \mathrm{mg} \mathrm{kg}^{-1}\right.$ day $^{-1}$ ) was administered twice daily for 7 days by continuous intravenous injection. Each drug dose was based on previously published studies in rodents (Shibata et al. 2002; Huisman et al. 2003; Manda et al. 2010; Pistell et al. 2010; Waring et al. 2010; Yang et al. 2010; du Plooy et al. 2011; Fontes et al. 2011; LledoGarcia et al. 2011; Wagner et al. 2011; Mak et al. 2013; Reyskens and Essop 2013a, b; Reyskens et al. 2013). The catheters were flushed with $0.3 \mathrm{ml}$ heparin $(50 \mathrm{IU} / \mathrm{ml})$ in PBS until the end of treatments. At time of euthanasia, preceding decapitation and tissue harvest, catheter patency was reverified by response to pre-euthanasia sedation $(100 \mathrm{mg} / \mathrm{kg}$ ketamine per $10 \mathrm{mg} / \mathrm{kg}$ xylazine). The brains were removed, the frontal cortex and the hippocampus were dissected on ice, and the tissue samples were stored at $-80^{\circ} \mathrm{C}$ until immunoblot analysis.

Immunofluorescence staining of tissue Tissue slides of paraffin-embedded tissue sections from hippocampus of male and female pig-tailed macaques (M. nemestrina) were prepared for immunofluorescent staining, as described previously (Akay et al. 2011a) . Briefly, glass slides containing paraffinembedded sections $(10 \mu \mathrm{m})$ were heated to $55^{\circ} \mathrm{C}$ for at least $30 \mathrm{~min}$, deparaffinized in Histoclear, and rehydrated with consecutive $100,95,90$, and $70 \%$ ethanol washes. Endogenous peroxidase activity was blocked with $3 \% \mathrm{H}_{2} \mathrm{O}_{2}$ in methanol and antigen unmasking was achieved with target retrieval solution at $95{ }^{\circ} \mathrm{C}$ for $1 \mathrm{~h}$. Tissue sections were blocked with $10 \%$ normal goat serum in phosphate-buffered saline solution (PBS). Mouse monoclonal antibodies to synaptophysin, MAP2, and rabbit polyclonal antibody to GFAP were used at empirically defined dilutions (synaptophysin at 1:500; MAP2 at 1:100; and GFAP at 1:80), and DAPI was used to stain nuclei. The tyramide amplification system was used to detect synaptophysin. Slides were mounted in Citifluor AF1, and for each specimen, five to ten randomly selected areas within the CA1-CA3 layer of the hippocampus were scanned along the $z$-axis to create $\mathrm{z}$ stack images at high magnification $(\times 600)$ by laser confocal microscopy on a Biorad Radiance 2100 equipped with Argon, Green $\mathrm{He} / \mathrm{Ne}$, Red Diode, and Blue Diode lasers (Biorad, Hercules, CA). Post-acquisition analysis was conducted using MetaMorph 6.0 (Universal Imaging, Downingtown, PA). Total intensity for synaptophysin, MAP2 and GFAP were determined by the measurement of integrated pixel intensity for synaptophysin, MAP2 or GFAP per $z$-stack image, where the integrated pixel intensity is defined as total pixel intensity per image times the area of pixels positive for synaptophysin, $\mathrm{MAP} 2$ or GFAP. Averages are expressed as mean $\pm \mathrm{SEM}$. All data was analyzed by Prism 5.0 software (GraphPad Software, San Diego, CA).

MAP2 cell-based ELISA A MAP2 cell-based ELISA was performed to quantify neuronal damage/death as previously described (White et al. 2011). Briefly, primary rat cortical neuroglial cultures plated in 96-well plates were fixed for 30 min with $4 \%$ paraformaldehyde in $4 \%$ sucrose at the conclusion of treatments. After blocking for $1 \mathrm{~h}$ with $5 \%$ normal goat serum in PBS, the plates were incubated with monoclonal MAP2 antibody overnight at $4{ }^{\circ} \mathrm{C}$, followed by washes with PBS with $0.1 \%$ Tween-20 (PBS-T). The plates were then incubated for $30 \mathrm{~min}$ with goat anti-mouse secondary antibody conjugated to beta-lactamase TEM-1 at room temperature (RT), washed with PBS-T, and incubated in the dark at RT for $1 \mathrm{~h}$ in fluorocillin green substrate. Fluorescence 
intensity was measured using a Fluoroskan Ascent fluorometer plate reader (Thermo Electron, Waltham, MA) with excitation at $485 \mathrm{~nm}$ and emission at $527 \mathrm{~nm}$.

Hand counting of MAP2-positive cells As a complementary method to MAP2 ELISA, neuronal survival was verified by hand counting of MAP2-positive neurons; $15 \mu \mathrm{M}$ propidium iodide was added to primary cortical neuroglial cultures grown on coverslips $15 \mathrm{~min}$ before the end of the treatments. The coverslips were washed once with PBS and fixed for 30 min with $4 \%$ paraformaldehyde in $4 \%$ sucrose, followed by blocking and permeabilization in $0.2 \% \mathrm{BSA}+0.1 \%$ Triton $\mathrm{X}-100$ in PBS for $1 \mathrm{~h}$ at RT. The coverslips were then washed twice with PBS and incubated with anti-MAP2 antibody $(1: 100)$ in normal antibody diluent for $2 \mathrm{~h}$ at RT. After two washes with PBS-T, the coverslips were incubated in a fluorescein isothiocyanate-conjugated goat anti-mouse IgG secondary antibody (1:200) for $30 \mathrm{~min}$ at RT. The coverslips were mounted on slides and alive/dead neurons were hand-counted based on MAP2 and PI-positive staining using a Nikon Eclipse E400 fluorescent microscope (Nikon Corp, Tokyo, Japan) equipped with Olympus DP70 digital camera (Olympus Corp, Tokyo, Japan). Live cells stain negative for PI, while dead cells retain it in their nuclei. The number of surviving MAP2-positive neurons will be positive for MAP2 and negative for PI staining. The percentage of MAP2positive cells \pm SEM were calculated from blinded counting of six fields at $\times 200$ in two adjacent vertical columns through the center of each coverslip, proceeding from top to bottom and bottom to top. For each condition, three coverslips were counted from two or more independent experiments.

Quantification of synaptophysin Cultures grown on coverslips, as described above, were immunofluorescently stained for synaptophysin (1:1,000), MAP2 (1:100), and DAPI. Tyramide amplification was used for the detection of synaptophysin. For each treatment condition, images of 10 randomly selected areas from three coverslips from three independent experiments were captured by fluorescence microscopy. Post-acquisition analysis was performed using NIH ImageJ program (V 1.36b, Bethesda, MD). Briefly, synaptophysin-positive puncta were detected by background subtraction and manual thresholding. The number of synaptophysin-positive puncta was determined by dividing the total number of puncta within a dendrite segment by the length of the segment.

Measurement of reactive oxygen species The superoxide indicator dihydroethidium (DHE, Invitrogen) was used to detect the presence of reactive oxygen species (ROS) in vitro. $3 \mu \mathrm{M}$ DHE was added to culture media 15 min prior to conclusion of treatments. Cells were then washed with PBS, fixed with $4 \%$ paraformaldehyde in $4 \%$ sucrose at RT for $8 \mathrm{~min}$, and stained for DAPI. The coverslips were then mounted on slides with CytoSeal and visualized using fluorescent microscopy, as described above. Post-acquisition analysis was performed using MetaMorph to determine the fluorescence intensity of DHE normalized to the area of DAPI signal.

Immunoblotting Whole cell extracts of rat tissue samples were prepared by homogenization in ice-cold tissue extraction buffer (50 mM Tris at $\mathrm{pH} 7.5,0.5 \mathrm{M} \mathrm{NaCl}, 1 \% \mathrm{NP}-40,1 \%$ SDS, $2 \mathrm{mM}$ EDTA, $2 \mathrm{mM}$ EGTA, $5 \mathrm{mM} \mathrm{NaF}, 0.4 \mathrm{mM}$ $\mathrm{Na}_{3} \mathrm{VO}_{4}, 1 \mathrm{mM}$ dithiothreitol (DTT), and 1:100 PI cocktail), followed by centrifugation at $12,000 \times g$ at $4{ }^{\circ} \mathrm{C}$ for $20 \mathrm{~min}$. Whole cell extracts of primary rat cortical cultures were prepared with ice-cold cell lysis buffer $(50 \mathrm{mM}$ Tris at $\mathrm{pH} 7.5$, $120 \mathrm{mM} \mathrm{NaCl}, 0.5 \% \mathrm{NP}-40,10 \mathrm{mM}$ EDTA, $0.4 \mathrm{mM}$ $\mathrm{Na}_{3} \mathrm{VO}_{4}, 100 \mathrm{mM}$ DTT, and 1:100 PI cocktail), followed by centrifugation at $14,000 \times g$ at $4{ }^{\circ} \mathrm{C}$ for $10 \mathrm{~min}$. The protein concentrations of the collected supernatants were determined using the Bradford method and $25-50 \mu \mathrm{g}$ of protein was loaded into each lane of a 4-12\% Bis-Tris gradient gel for separation. A broad range molecular weight ladder was run on each gel. Subsequent to separation, proteins were transferred onto PVDF membranes, and blocked in Tris-buffered saline with $0.1 \%$ Tween- 20 (TBS-T) and $5 \%$ bovine serum albumin (BSA) for $1 \mathrm{~h}$ at RT. The membranes were incubated with the primary antibodies in TBS-T with $5 \% \mathrm{BSA}$ at $4{ }^{\circ} \mathrm{C}$ overnight, washed with TBS-T, followed by incubation with corresponding HRP-conjugated secondary antibodies. The membranes were developed using SuperSignal West Dura extended duration substrate. Loading controls were obtained by staining the membranes and the gels with the Biosafe Coomassie Stain for $20 \mathrm{~min}$, followed by destaining with deionized water for $30 \mathrm{~min}$. For densitometric analysis, autographs were scanned into Adobe Photoshop (Adobe Systems, San Jose, CA) and regions of interests (ROI) of equal size were determined for each band. The pixel intensities of ROIs were quantified using the NIH ImageJ program (V 1.36b, Bethesda, MD). Target band intensities were normalized to gel and membrane coomassie stain controls to account for protein degradation and loading discrepancies, and the normalized target band intensities were used to quantify fold changes over controls.

Quantitative reverse transcription polymerase chain reaction The expression of HO-1 and NQO-1 genes in rat neuroglial cells was quantified by quantitative reverse transcription polymerase chain reaction (qRT-PCR). Custom TaqMan ${ }^{\circledR}$ Gene Expression Assays were purchased from Applied Biosystems for the genes: NQO-1 (Rn00566528_m1) and HO-1 (Rn01536933 m1). Approximately $5 \mathrm{ng}$ cDNA was used per reaction. StepOne ${ }^{\mathrm{TM}}$ Software v2.0 was used to construct the experimental protocol and the qRT-PCR took place in the StepOne Real-Time PCR System (Applied Biosystems, Carlsbad, CA). 
Data was normalized using both $\beta$-actin (Rn00667869 m1) and 18S (Hs99999901_s1) and was analyzed according to the $\Delta \Delta \mathrm{C}_{\mathrm{T}}$ method. All samples were run in triplicate from three biological replicates.

Statistical analysis All data was analyzed by Prism 5.0 software (GraphPad Software, San Diego, CA). Values are expressed as mean \pm SEM, and values of $p<0.05$ were considered significant for all statistical analyses performed.

\section{Results}

Antiretroviral drugs lead to neuronal damage in vivo

cART-induced peripheral neuropathy is well documented (Power et al. 2009), and it is likely that cART triggers similar damage to neurons in the CNS. To determine the neurotoxic potential of cART in the CNS, we assessed the effects of an ARV regimen on the expression of synaptophysin and MAP2, indicators of synaptic damage and neuronal loss, respectively, utilizing post-mortem tissue from a well-characterized SIV/ pigtail macaque model of HIV CNS disease, which was designed to address the efficacy of CNS penetrant antiretroviral therapy in reducing viral load in the CNS (Zink et al. 2010). In this study, animals infected with SIV either received no cART or received early cART treatment that included tenofovir (NRTI), atazanavir (PI), saquinavir (PI), and L-870812a (integrase inhibitor) 12 days after the virus inoculation. Without cART, $90 \%$ of animals develop neurologic disease within 3 months postinoculation (p.i.). By contrast, animals receiving cART do not develop SIV encephalitis. Rather, they show a rapid reduction in their plasma and CSF viral load followed by continued suppression of SIV replication with maintenance of CD4+ T cell counts until elective euthanasia around day 160 p.i. Additionally, cARTtreated animals do not exhibit any outward signs of neurological deficits. Further, our quantitative immunofluorescent analysis of hippocampal tissue sections revealed reduced astrogliosis in the hippocampus of SIV-infected, cART-treated animals (SIV $(+) /$ cART), compared with SIV-infected macaques that did not receive cART $((\operatorname{SIV}(+) /$ placebo)) (Fig. 1b). However, we observed statistically significant decreases in synaptophysin expression in the hippocampi of the SIV $(+) /$ cART group, compared with that in either the uninfected or the SIV $(+) /$ placebo group (Fig. 1a, d). In addition, examination of the expression of a second marker of synaptodendritic integrity, calmodulin kinase II (CaMKII), by immunoblotting showed that CaMKII levels were significantly lower in the frontal cortex in the SIV $(+) /$ cART macaques than in their SIV $(+) /$ placebo counterparts (Fig. 1e, f). CaMKII is highly expressed in neurons of macaque hippocampus and frontal cortex, whereas its expression in other cell types, including microglia, infiltrating macrophages, and multinucleated giant cells, is minimal; thus, the differences in CaMKII expression in frontal cortex of the animals from our experimental groups are neuron specific (Gupta et al. 2010). Our results demonstrate synaptic injury in the presence of cART despite effective control of SIV replication in the periphery and CNS. Interestingly, we did not observe changes in MAP2 fluorescence in the hippocampus of infected and/or cARTtreated animals compared with untreated/uninfected animals (Fig. 1a, c).

As studies of uninfected, cART-treated macaques have not been performed to determine the contribution of cART to neuronal damage independent of SIV infection, we administered combinations of ARVs intravenously to adult rats. In patients, initial cART usually includes two nucleoside/NRTIs in combination with a nNRTI or with a PI boosted with a low dose of a second PI, ritonavir (Rit). Thus, we used zidovudine (AZT), an NRTI, along with two PIs, saquinavir (Saq) and Rit, at doses based on previously published pharmacokinetic studies of these ARVs (Busidan and Dow-Edwards 1999; Kageyama et al. 2005; Pistell et al. 2010). In agreement with previous studies (Waring et al. 2010), the animals showed no overt signs of distress during the course of the treatment. However, via immunoblotting, we observed decreases in hippocampal synaptophysin expression in cART-treated rats compared with vehicle-treated rats (Fig. 1g), complementing our findings of synaptic damage in $\operatorname{SIV}(+) / c$ ART macaques and further supporting a role for ARV-associated neuronal injury in the CNS. Of note, we also detected decreases in MAP2 levels in the cART-treated rat hippocampus (Fig. 1g), which likely reflects the acute drug toxicity in this treatment paradigm, compared to the subtler synaptic injury observed in the cART-treated macaque brain.

Antiretroviral compounds in therapeutically relevant combinations are neurotoxic in vitro

The only primary neural cell-type previously studied for ARV toxicity was dorsal root ganglia neurons in models of peripheral neuropathy (Werth et al. 1994). Here, to expand our studies on ARV neurotoxicity in the CNS, we used primary rat cortical neuroglial cultures aged 21 DIV (O'Donnell et al. 2006; Wang et al. 2007; White et al. 2011). We first evaluated the neuronal viability in response to increasing concentrations of AZT, Rit, or Saq. We based the range of doses on reported plasma and CSF levels of ARVs (Wynn et al. 2002; Anthonypillai et al. 2004). Importantly, animal studies predict brain parenchymal levels to be equal to or greater than CSF levels (Anthonypillai et al. 2004; Anderson and Rower 2010). At $48 \mathrm{~h}$ posttreatment with individual ARVs, Rit and Saq both led to dose-dependent decreases in MAP2-positive cells, as determined via hand counting (Fig. 2a, b, respectively). These results were confirmed with a cell-based MAP2 ELISA, which accurately reflects neuronal numbers, as well as 

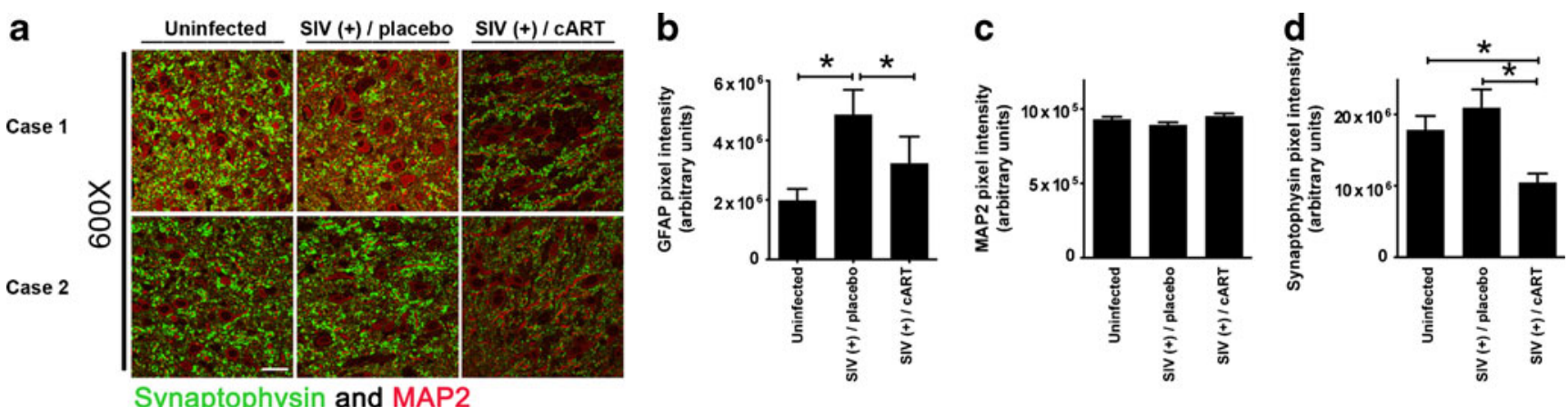

e

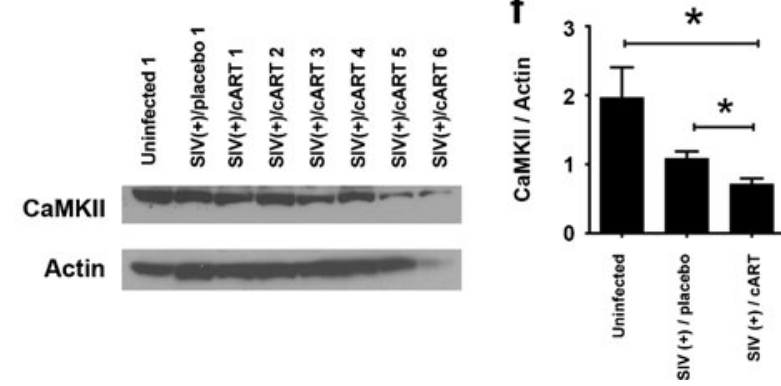

Fig. 1 Antiretroviral drugs induce neuronal damage in vivo. a-d Formalin-fixed, paraffin-embedded tissue sections from hippocampus of pigtailed macaques that were either uninfected $(n=6)$, SIV infected but not cART treated $(n=7)$, or SIV infected and treated with cART (tenofovir, atazanavir, saquinavir, and L-870812a; $n=4)$ were prepared for immunofluorescent analysis and were triple labeled for MAP2 (red), synaptophysin (green), and GFAP. Sections were visualized by laser confocal microscopy and images were quantified for MAP2, synaptophysin and GFAP expression. a Representative composite images of two cases per group which were stained with MAP2 and synaptophysin are shown. Scale bar $=30 \mu \mathrm{m}$. b Quantification shows the resolution of GFAP immunoreactivity in SIV $(+) / c$ ART group, compared with $\operatorname{SIV}(+) /$ placebo group (one-way ANOVA, ${ }^{*} p<0.05$ ). No changes were observed in MAP2 expression between groups (c), but there were statistically significant decreases in synaptophysin

neuronal damage (Fig. 2d, e) (White et al. 2011). Furthermore, neuronal damage and death induced by Rit and Saq was time dependent, as seen in Fig. $2 \mathrm{~g}$, where the primary cortical cultures were exposed to individual ARVs for up to 8 days. Additionally, both Rit and Saq led to dose-dependent decreases in synaptophysin expression after $16 \mathrm{~h}$ of treatment, well before the loss of MAP2-positive cells occurred (Fig. 2h, i). However, AZT did not lead to decreases in MAP2-positive cells, MAP2 fluorescence, or synaptophysin expression (Fig. 2c, f, and data not shown, respectively). Next, we treated primary cortical cultures with drug combinations that included AZT, Rit, and Saq, alone or in combinations, for $48 \mathrm{~h}$, to assess acute neurotoxicity. As seen in Fig. 2j, k, combination treatments that included Rit induced statistically significant neuronal damage/death, which were preceded by loss of synaptophysin detected at $16 \mathrm{~h}$ posttreatment (Fig. 2l). Additionally, we observed similar levels of neuronal damage in cortical cultures treated with a combination of $\mathrm{d} 4 \mathrm{~T}$, Rit, and Saq (not shown). However, as d4T is no longer prescribed in most developed countries and the AZT/Rit/Saq combination g

Synaptophysin
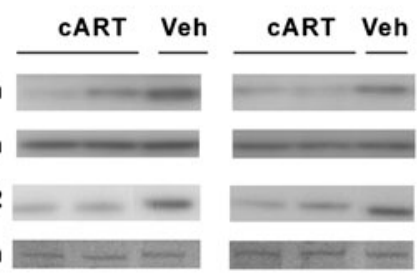

immunoreactivity (d) in $\operatorname{SIV}(+) /$ cART group, as compared with $\operatorname{SIV}(+) /$ untreated and uninfected groups (one-way ANOVA, ${ }^{*} p<0.05$, ns not significant). e, f Fresh-frozen tissue sections from the frontal cortex of pig-tailed macaques that were either uninfected $(n=3)$, SIV infected but not cART treated $(n=6)$, or SIV infected and cART treated $(n=6)$ were used for standard protein extraction and subsequent immunoblotting for the expression of CaMKII. Actin was used as a loading control. A representative immunoblot is shown. Quantification shows statistically significant decreases in CaMKII in the cART-treated group, as compared with the uninfected group or the $\operatorname{SIV}(+) /$ untreated group (one-way ANOVA, $* p<0.05)$. g Whole cell lysates prepared from hippocampus of rats treated for 7 days with AZT/Rit/Saq $(n=4)$ or vehicle $(n=2)$ were immunoblotted for synaptophysin and MAP2. A band from the coomassie blue staining is included to control for equal loading and protein degradation

reflects the first considered/prescribed combination in initial treatment plans, we focused on AZT/Rit/Saq combination treatments for further experiments. Next, we determined that treatment with either Rit or Saq, alone or in combination with AZT, lead to the activation of the $\mathrm{Ca}^{2+}$-activated death protease, calpain, as evidenced by the increase of calpain-cleaved spectrin observed in these cultures (Fig. 3). Interestingly, none of the treatment combinations caused increases in the cleaved, and therefore active, form of caspase-3 (Fig. 3), suggesting that neuronal death observed in our model may be a necrotic cell death rather than an apoptotic cell death. Together the findings presented here suggest that combination ARV treatments that include PIs are toxic to cortical neurons in vitro.

Combination antiretroviral drug treatments induce oxidative stress in neurons

An extensive number of studies have linked ARVs, especially PIs, to oxidative stress (Touzet and Philips 2010). To determine whether neurons undergo oxidative stress when exposed 
to ARVs, we used dihydroethidium (DHE) as a marker for the presence of ROS (Rodriguez-Pallares et al. 2009). We included tert-butyl hydroperoxide, an organic pro-oxidant, at $100 \mu \mathrm{M}$, as a positive control. As seen in Fig. 4a and quantified in Fig. 4d, Rit/Saq and AZT/Rit/Saq combination drug treatments for $6 \mathrm{~h}$ lead to ROS accumulation in cortical cultures, whereas ROS levels in vehicle-treated cultures were comparable to those in untreated cultures.

The cortical cultures generated for our studies contain $85-90 \%$ neurons and $10-15 \%$ astrocytes (Fig. 4b); thus, we also determined the presence of ROS in pure neuronal and pure astrocytic cultures exposed to individual ARVs. As seen in Fig. 4c and quantified in Fig. 4e, Rit led to an early and sustained ROS production in pure neuronal cultures, whereas Saq-induced ROS accumulation occurred $24 \mathrm{~h}$ after treatment. The transient ROS accumulation induced by AZT was resolved at $24 \mathrm{~h}$ posttreatment. Conversely, in pure astrocytic cultures, none of the three ARVs examined induced an appreciable sustained ROS generation, and only Rit caused a transitory ROS accumulation (Fig. 5a, b). These data further suggest that the ARV-induced ROS production observed in primary cortical neuroglial cultures are occurring in neuronal cell populations.

Endogenous antioxidant response activation by combination antiretroviral drugs

Oxidative stress in cells triggers the transcriptional induction of oxidative stress responsive genes through the activation of the endogenous antioxidant response element in their promoter regions (Chen and Kong 2004). Thus, we determined the effect of ARVs on two such genes, NQO-1 and HO-1. By qRT-PCR, we observed increased mRNA levels of both NQO-1 and HO-1 in cultures treated with any of the tested combinations of ARVs, when compared with the levels in untreated cultures (Fig. 6a, b, respectively), with the most striking increases observed in Rit/Saq-treated cultures. To determine whether the changes in the mRNA levels were reflected in protein levels, we used immunoblotting to examine protein levels of NQO-1 and HO-1. While we observed increases in NQO-1 and HO-1 protein levels at $16 \mathrm{~h}$ (Fig. 6c), the changes were more robust in cultures treated for $48 \mathrm{~h}$ (Fig. 6d). Further, in vivo, we detected increased levels of HO-1 protein in hippocampal lysates from cART-treated rats (Fig. 6e, f). Interestingly, NQO-1 levels were not increased in cART-treated rat hippocampus (Fig. 6e). Of note, we did not observe an antioxidant response in ARV-treated pure astrocytic cultures (Fig. 5c-e). These data collectively suggest an activation of HO-1, as part of the endogenous antioxidant response, following ARV treatments in neurons.
Monomethyl fumarate protects against ROS generation and induces activation of the endogenous antioxidant response

NQO-1 and HO-1 are targets of the fumaric acid ester, dimethyl fumarate (DMF), and of its hydrolyzed and active metabolite, MMF (Linker et al. 2011). Our recent study (Cross et al. 2011) has shown that DMF and MMF reduce neurotoxin release from HIV-infected macrophages through induction of HO-1. We reasoned that augmented or earlier induction of NQO-1 and HO-1 in neurons by pretreatment with the active metabolite, MMF, would provide protection in our in vitro model of ARV-induced neurotoxicity through an antioxidant effect. We first determined that MMF at 30 $100 \mu \mathrm{M}$ was not toxic in neuronal cultures, as determined by MAP2 ELISA (data not shown). Next, we preincubated primary cortical cultures with $100 \mu \mathrm{M}$ MMF for $30 \mathrm{~min}$ before adding either Rit/Saq or AZT/Rit/Saq. Preincubation with MMF blocked ROS generation induced by ARV treatments lasting 6 (Fig. 7a), 16, and $48 \mathrm{~h}$ (data not shown). Quantification showed ROS levels in cultures preincubated with MMF to be comparable to those detected in untreated and vehicle-treated cultures (Fig. 7b). Furthermore, we observed an early increase in HO-1 protein levels at $4 \mathrm{~h}$ in cultures preincubated with MMF, whether or not pretreatment was followed by ARV treatment (Fig. 7c). This trend was sustained at $16 \mathrm{~h}$ after combination ARV treatment, as well. Next, we determined whether augmenting the endogenous antioxidant response by MMF blocks MAP2 loss induced by combination ARV treatments. As detected by the cellbased MAP2 ELISA, MMF blocked neuronal damage/death in cultures treated with Rit/Saq and AZT/Rit/Saq for $72 \mathrm{~h}$ (Fig. 7d). Finally, pretreatment of the cultures with an HO-1 inhibitor, SnMP (Zhao et al. 2004), reversed the MMFinduced decreases in ROS accumulation (Fig. 7e), suggesting that MMF protection occurs through its augmentation of $\mathrm{HO}$ 1 expression.

\section{Discussion}

Virus-related factors, such as resistant virus species and persistent viral DNA in the CNS, may contribute to the persistence of HAND in the post-cART era. One recent focus in HIV neurovirology is the development of ARVs with greater CNS penetrance (Letendre et al. 2008; Marra et al. 2009; Tozzi et al. 2009; Edén et al. 2010; Heaton et al. 2010; Garvey et al. 2011; Smurzynski et al. 2011). However, potential ARV toxicity in the CNS remains largely unexplored. Our study supports a possible contribution of the ARVs themselves to neuronal and synaptic damage observed in patients with HAND. 

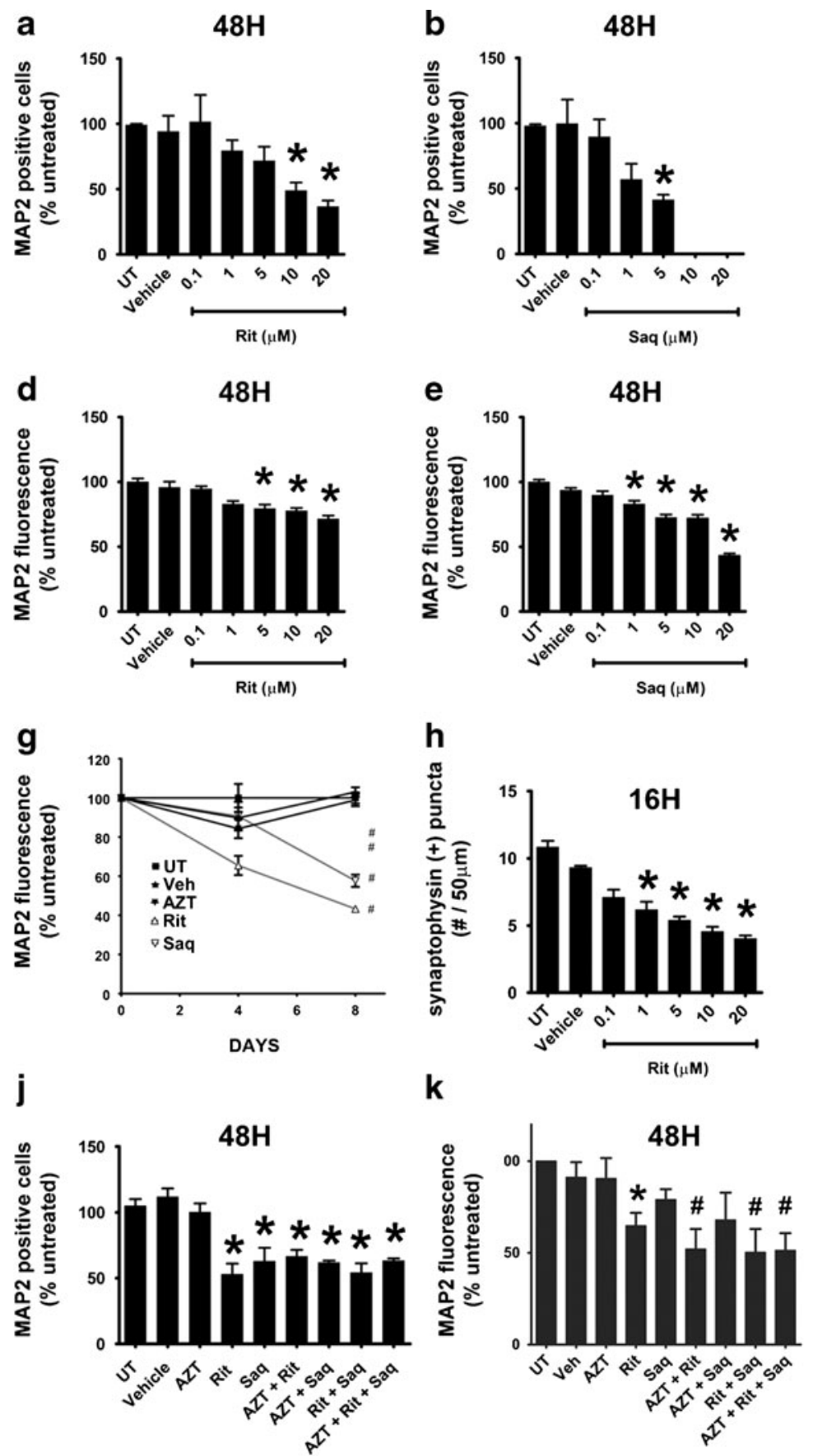
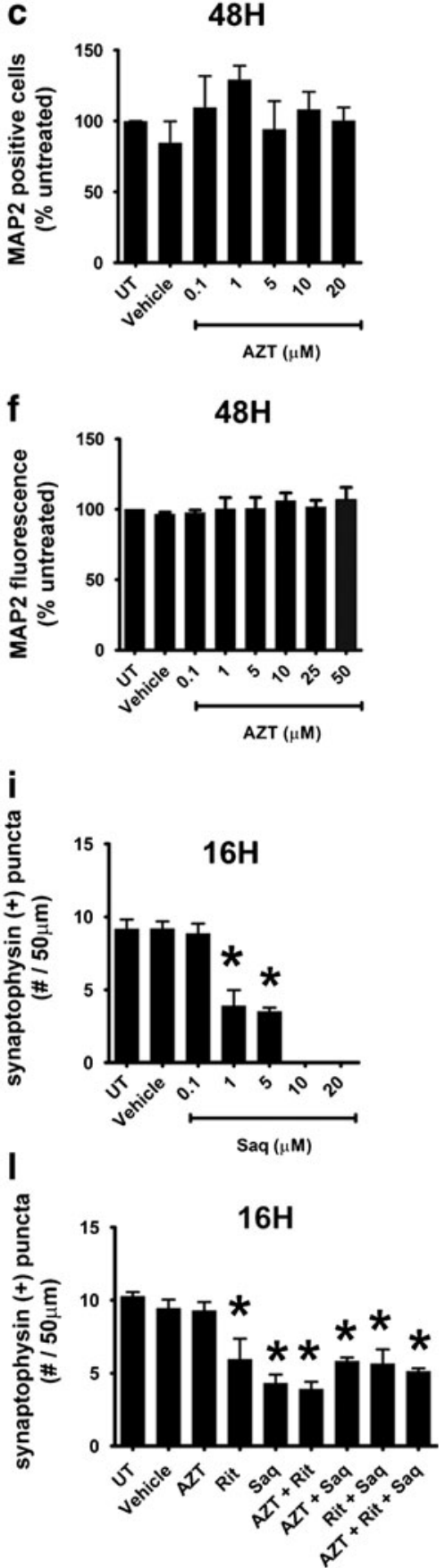

Fig. 2 Therapeutically relevant combination antiretroviral drug treatments are neurotoxic in vitro. a-c Primary rat cortical neuroglial cultures aged 21 days in vitro $(D I V)$ on coverslips were exposed to Rit (a), Saq (b), or AZT (c) at increasing doses for $48 \mathrm{~h}$, followed by hand counting for MAP2-positive cells ( $n=3$; vehicle, $0.04 \%$ DMSO; $* p<0.05$, one-way ANOVA with post-hoc Newman-Keuls). d-f 21DIV primary neuroglial cultures grown in 96-well plates were treated with increasing doses of Rit (d), Saq (e), or AZT (f) for $48 \mathrm{~h}$, followed by MAP 2 cell-based ELISA $(n=2$; vehicle, $0.04 \%$ DMSO; * $p<0.05$, one-way ANOVA with post-hoc Newman-Keuls). g Primary neuroglial cultures were treated with AZT $(25 \mu \mathrm{M})$, Rit $(10 \mu \mathrm{M})$, or Saq $(1 \mu \mathrm{M})$ at day zero. Ninety percent of the media was changed with conditioned media supplemented with a fresh drug stock every 2 days, and cultures were analyzed by MAP 2 cell-based ELISA at days 4 and $8(n=2$; vehicle, $0.04 \%$ DMSO; $* p<0.05$, one-way ANOVA with post-hoc Newman-Keuls). h, i Cultures grown on coverslips were exposed to the indicated treatments and synaptophysin-positive puncta were determined $(n=3$; vehicle, $0.04 \%$ DMSO; $* p<0.05$, one-way ANOVA with post-hoc Newman-Keuls). j, k Primary neuroglial cultures were exposed to the indicated drug combinations (AZT, $25 \mu \mathrm{m}$; Rit, $10 \mu \mathrm{m}$; Saq, $1 \mu \mathrm{m}$ ) for $48 \mathrm{~h}$, followed by hand counting for MAP2-positive cells (j) or MAP2 cell-based ELISA (k) ( $n=2$; vehicle,: $0.04 \%$ DMSO; $* p<0.05$; $\# p<0.01$, one-way ANOVA with post-hoc Newman-Keuls). I Primary neuroglial cultures that were exposed to the indicated drug combinations (AZT, $25 \mu \mathrm{m}$; Rit, $10 \mu \mathrm{m}$; Saq, $1 \mu \mathrm{m})$ for $16 \mathrm{~h}$ were analyzed for synaptophysin-positive puncta $(n=2$; vehicle, $0.04 \%$ DMSO; $* p<0.05$, one-way ANOVA with post-hoc Newman-Keuls) 


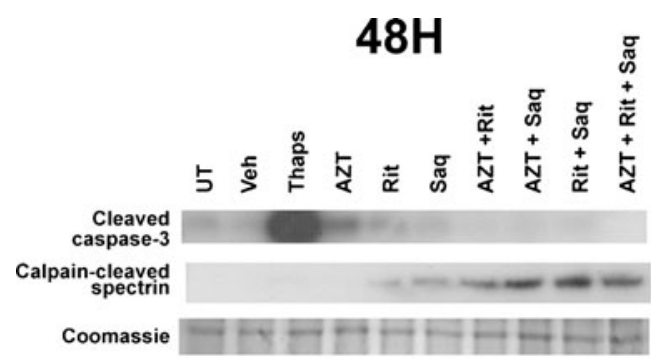

Fig. 3 Activation of Calpain in antiretroviral drug-treated neurons. Whole cell lysates were prepared from neuroglial cultures treated with the indicated single or combination drugs (AZT, $25 \mu \mathrm{m}$; Rit, $10 \mu \mathrm{m}$; Saq, $1 \mu \mathrm{m})$, or with Thapsigargin $(1 \mu \mathrm{M})$ as a positive control, for $48 \mathrm{~h}$. Calpain activation was assessed using an antibody to detect the accumulation of calpain-cleaved spectrin and an antibody raised against the cleaved and active form of caspase-3 was used for detection of caspase activity. A band revealed by coomassie staining of the gel was used as a loading control $(n=2$; vehicle, $0.04 \% \mathrm{DMSO})$

In this study, we show cART-induced synaptophysin loss, indicative of synaptic injury in two animal models. In our first model, in an in vivo model of SIV-infected pig-tailed macaques, we report decreased synaptophysin and CaMKII levels in the SIV $(+) /$ cART group compared with uninfected or SIV $(+) /$ placebo groups, indicating synaptodendritic damage. Interestingly, MAP2 levels did not change significantly across groups, which may be because of the relatively short duration of infection in this retrospective study cohort. While these data demonstrate potential effects of cART drugs in the presence of viral infection, there are three variables to consider in interpretation of these findings: (1) the time to euthanasia from the start of the experiments is different between $\operatorname{SIV}(+) /$ placebo and SIV $(+) /$ cART groups, (2) persistent viral DNA in the CNS of SIV $(+) /$ cART group, and (3) the lack of SIV(-)/ cART group. It is not yet known whether brain SIV DNA is replication competent. As we utilized post-mortem samples obtained from a cohort of macaques enrolled in a previous study addressing the efficacy of CNS penetrant cART in reducing viral loads in the CNS, further experiments that include an additional control group receiving cART but not inoculation with SIV will be instrumental to more clearly determine the contribution of viral DNA and cART to synaptic damage in this model.

In the second in vivo model presented here, adult rats received a therapeutically relevant combination of ARVs (NRTI+PI+Rit boost). In the small number of studies where pharmacokinetics and effects of ARVs in the CNS were examined, pathological read-outs of neuronal damage, such as MAP2 or synaptophysin loss, were not determined (Huisman et al. 2003; Anthonypillai et al. 2004; Anthonypillai et al. 2006). Synaptic injury is a known indicator of neuronal damage and dysfunction in various neurodegenerative diseases, including HAND (Gupta et al. 2010) and synaptodendritic injury persists in HIV-infected individuals in the post-cART era (Xu and Ikezu 2009). We observed decreases in synaptophysin and MAP2 protein levels in the hippocampus in response to ARV administration over 7 days. Thus, our model demonstrates that ARV-associated neurotoxicity warrants consideration in developing therapeutic regimens for HIV-infected patients.

We also show that the PIs, Rit and Saq, alone or in combinations with the NRTI, AZT, induce oxidative stress, and neuronal damage/death in primary cultures at clinically relevant doses. Previous studies examined ARV-induced toxicity in cell lines, and Robertson et al. have provided the first evidence for ARV-induced neurotoxicity in primary rat neurons (Robertson et al. 2012). Here, we provide further evidence that PI-induced oxidative stress and neuronal death in primary neurons can be blocked by the activation of the endogenous antioxidant response. Interestingly, in our experimental paradigm, the NRTI, AZT, neither induced neuronal damage/death by itself, nor augmented PI-induced damage/ death when used in combination. We observed similar effects from a combination using another NRTI, stavudine (d4T; not shown). In agreement with our observations, a previous study presented similar findings, specifically that neither AZT nor d4T inhibited cell growth or neurite regeneration in PC-12 cells after long-term drug exposure (Cui et al. 1997). It should be noted that NRTIs are unequivocally tied to peripheral neuropathy, where the underlying pathology is mitochondrial toxicity and oxidative stress. However, NRTIs affect only certain cell populations, as they are formulated as pro-drugs and, to become active, need to be phosphorylated by two kinases, thymidine kinase 1 and 2 (TK1 and TK2), and the expression of the cytoplasmic TK1 is cell cycle dependent (Bazzoli et al. 2010). Thus, in our model utilized to study postmitotic neurons, AZT is most likely not converted to its active form, and thus does not contribute to neuronal damage/death. It is of note that our in vitro model of ARV-induced neurotoxicity utilized primary neuroglial cultures rather than cell lines. Primary cells are untransformed, and therefore more accurately reflect and predict ARV-associated effects occurring in the brains of patients on cART than would immortalized cell lines. The molecular pathways we investigated in this study are highly conserved from yeast to human cells; thus, the results obtained here in cells of rodent origin are likely conserved in human cells as well.

The drug concentrations used in this study are based on the plasma and CSF levels reported by various in vitro and in vivo studies(Huisman et al. 2003). As reported in such studies, AZT can be detected in the CSF at concentrations that are similar to those measured in the plasma (Wynn et al. 2002). Contrarily, as backed by various in vitro and in vivo studies (Wynn et al. 2002), both Rit and Saq are predicted to have limited CNS penetrance due to the strong tendency of these drugs to bind plasma protein because of their lipophilic nature 
Fig. 4 Combination antiretroviral drug treatments induce oxidative stress in neurons. a, d Cortical neuroglial cultures grown on coverslips were treated for $6 \mathrm{~h}$ with the indicated drugs (AZT, $25 \mu \mathrm{m}$; Rit, $10 \mu \mathrm{m}$; Saq, $1 \mu \mathrm{m}$ ) and the presence of ROS was detected by DHE staining (red fluorescence). a The images were captured with epifluorescent microscopy with uniform settings. d Quantification of DHE fluorescence was generated by measurement of DHE pixel intensity per DAPI area $\left(n=3 ;{ }^{*} p<0.05 ; \# p<0.01\right.$, one-way ANOVA, post-hoc Newman-Keuls). b Cortical neuroglial cultures grown on coverslips for 21 days and exposed to the indicated treatments (AZT, $25 \mu \mathrm{m}$; Rit,

$10 \mu \mathrm{m}$; Saq, $1 \mu \mathrm{m})$ were immunofluorescently labeled for MAP2 (green) and GFAP (red). Note that the cultures are enriched for neurons; c 21 DIV pure cortical neuronal cultures grown on coverslips were treated with AZT $(25 \mu \mathrm{m})$, Rit $(10 \mu \mathrm{m})$, or Saq $(1 \mu \mathrm{m})$ for 2,12 , or $24 \mathrm{~h}$. The presence of ROS was detected by DHE staining (red fluorescence) and MAP2 was used to stain neurons (green fluorescence). e The images captured with confocal microscopy with uniform settings were analyzed for DHE pixel intensity per DAPI area $\left(n=3 ;{ }^{*} p<0.05\right.$, one-way ANOVA, post-hoc NewmanKeuls) a
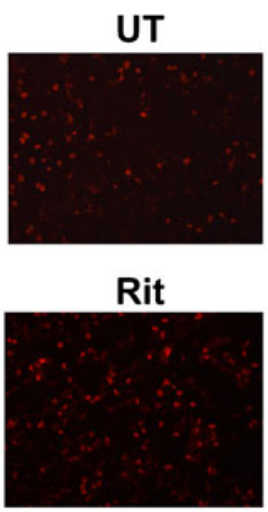

UT
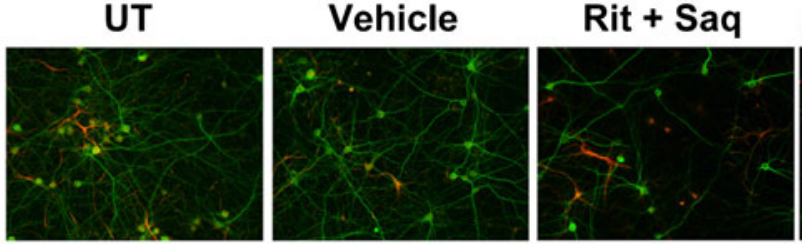

C

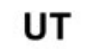

Vehicle

AZT

Rit
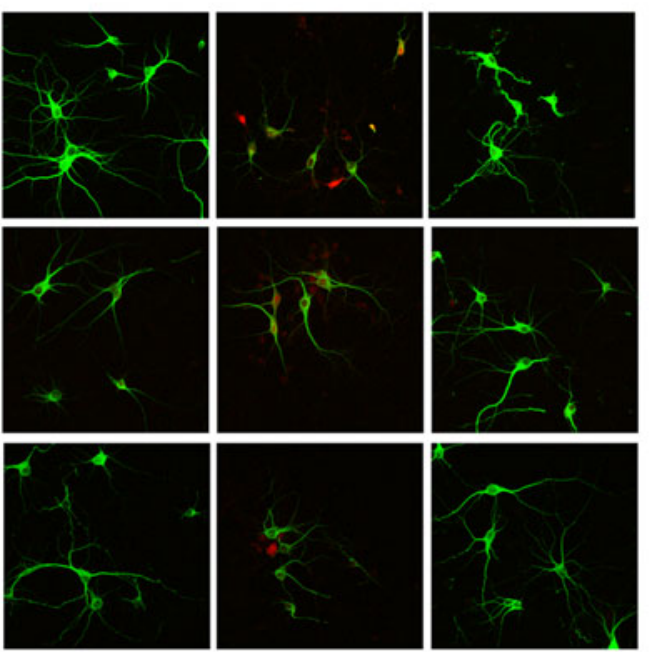

d

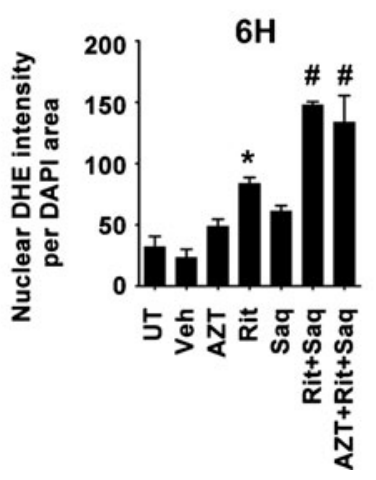

$2 \mathrm{H}$

e

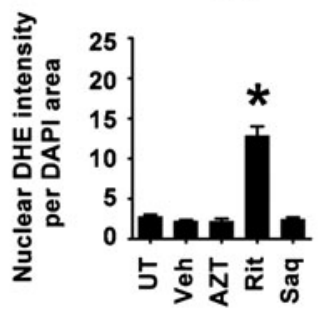

$12 \mathrm{H}$

$24 \mathrm{H}$

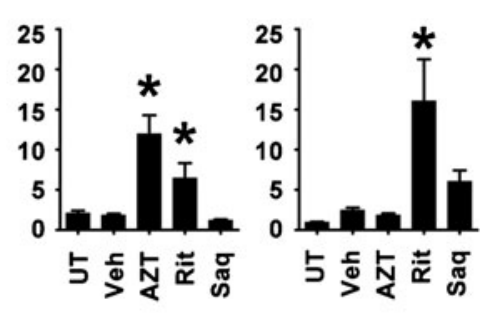

and pharmacokinetic properties. However, a comprehensive study conducted in an in situ guinea pig model suggests that Rit can achieve high concentrations in choroid plexus and parenchyma through diffusion via the choroid plexus; in fact, the levels of Rit were comparable to levels measured in plasma (Anthonypillai et al. 2004). Furthermore, the study showed that, surprisingly, the CSF levels of Rit were lower than the levels measured in the choroid plexus and the parenchymal compartments. Thus, the authors concluded that CSF concentrations of Rit may not necessarily reflect the 
Fig. 5 Antiretroviral drugs do not induce an endogenous antioxidant response in astrocytes. a Pure astrocytic cultures grown on coverslips were treated with AZT $(25 \mu \mathrm{m})$, Rit $(10 \mu \mathrm{m})$, or Saq $(1 \mu \mathrm{m})$ for 2,12 , or $24 \mathrm{~h}$. The accumulation of ROS was detected by DHE staining (red fluorescence) and GFAP was used to label astrocytes (green fluorescence). b The images captured with confocal microscopy with uniform settings were analyzed for DHE pixel intensity per DAPI area $(n=3 ; * p<0.05$, One-Way ANOVA, post-hoc NewmanKeuls). c-e Whole cell lysates from pure astrocytic cultures treated with the indicated drugs (AZT, $25 \mu \mathrm{m}$; Rit, $10 \mu \mathrm{m}$; Saq, $1 \mu \mathrm{m})$ for $16 \mathrm{~h}$ were immunoblotted for HO-1 and NQO-1. Representative blots from three independent experiments are shown in (c). Coomassie staining of gels were used as loading controls and fold changes over untreated lysates were determined. The quantification of HO-1 and NQO1 band intensities from three independent experiments is shown in (d) and (e) $(n=3$; ${ }^{*} p<0.05$, one-way ANOVA, posthoc Newman-Keuls)

a

UT

$2 \mathrm{H}$

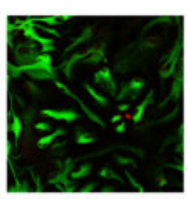

$12 \mathrm{H}$

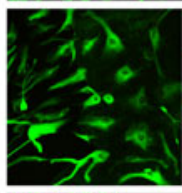

24H

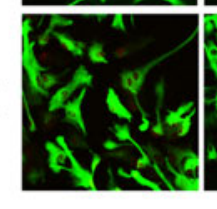

\section{Veh}

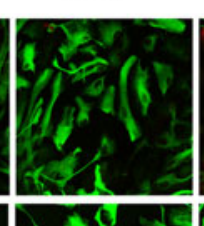

AZT

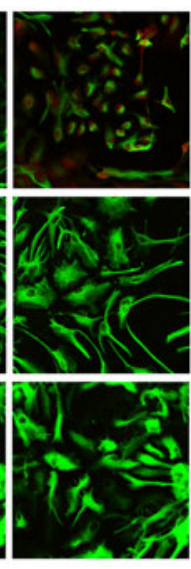

Rit

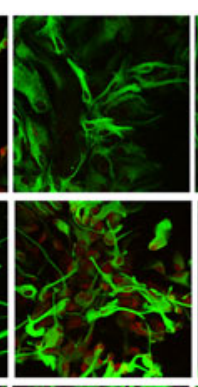

Saq
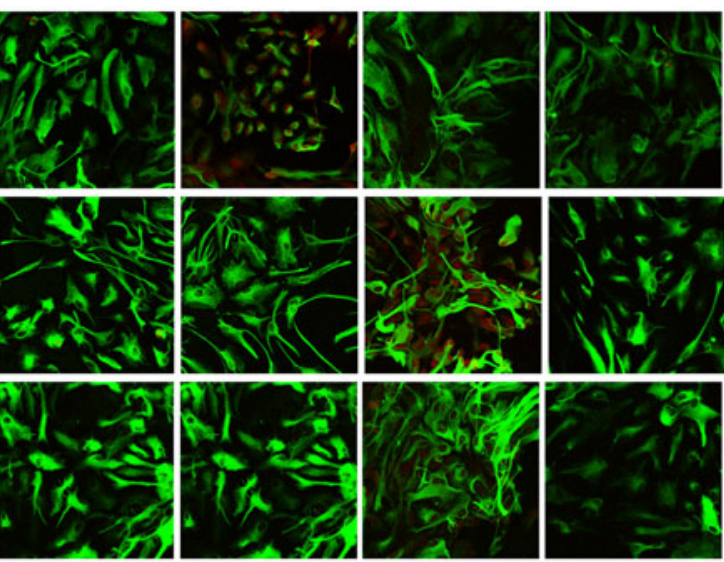

b

$2 \mathrm{H}$

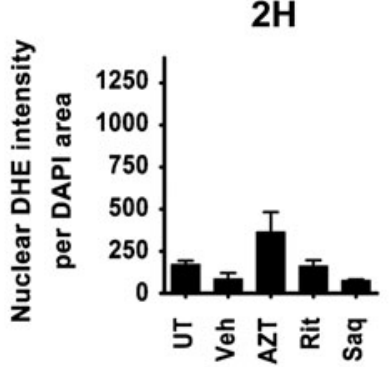

$12 \mathrm{H}$
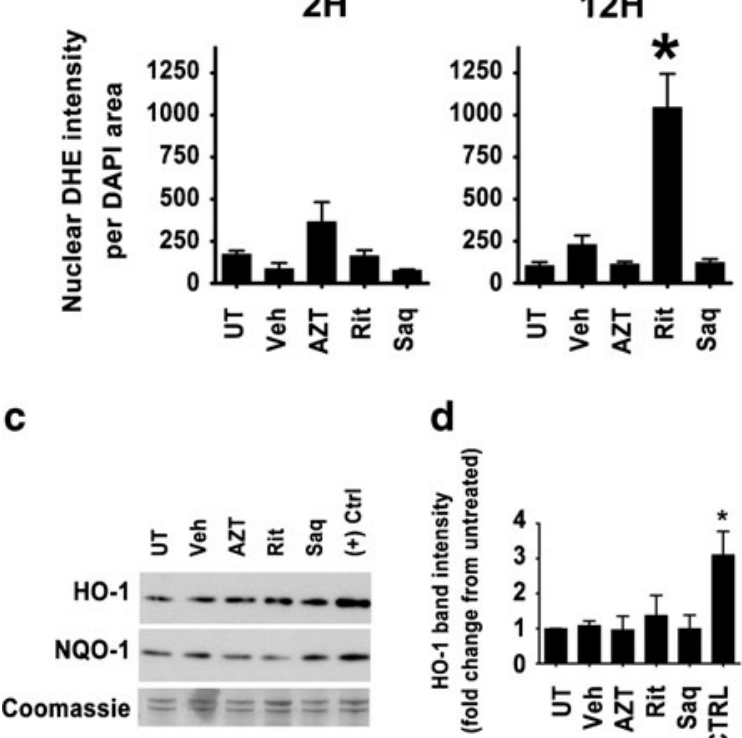

d

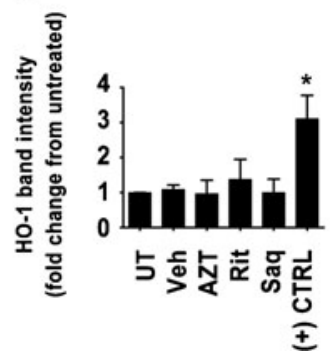

e

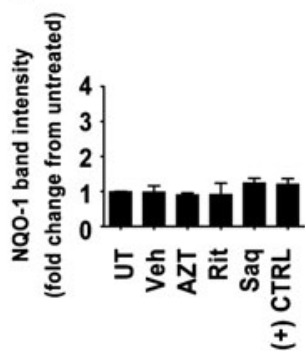

parenchymal levels, which are indeed a better indicator of effective drug levels in the CNS. Rit and Saq concentrations in our experiments fall well within plasma ranges reported in patients receiving cART.

Evidence of oxidative stress has long been associated with the most severe forms of HAND (Ngondi et al. 2006; Mielke et al. 2010). Interestingly, despite systemic control of viral replication in cART-treated patients, markers of oxidative stress, such as elevated levels of lipid and protein oxidation, are still detectable in the brains of these individuals (Ances et al. 2008). Of note, oxidative stress is one of the underlying mechanisms involved in NRTI and PI-induced toxicity in the periphery. Our data suggest that the sustained ROS accumulation in neurons due to prolonged exposure to ARVs might induce the oxidative stress associated with cART-induced toxicity in the CNS, leading to the observed changes in synaptophysin levels and the subsequent neuronal death.
Our data also suggest that astrocytes do not show neither ROS accumulation nor the endogenous antioxidant response in vitro. We also observe that astrogliosis in SIV-infected animals is resolved in cART-treated animals. While these findings suggest that astrocytes may not be highly impacted by ARVs in the short term, it is possible that prolonged exposure to ARVs might overwhelm astrocytes, which help buffer ROS accumulation in neurons under normal conditions, precipitating further neuronal damage.

We further show that the ARV drug-induced effects observed in this study can be blocked by MMF via activation of an endogenous antioxidant response. Cells respond to oxidative stress by activating the transcription of a subset of genes in an effort to clear excess ROS within the cell. Among these genes are NQO-1 and HO-1 (Chen and Kong 2004). Here, we show increased levels of NQO-1 and HO-1 mRNA and protein in ARV-treated neuronal cultures. Additionally, pretreatment of cultures with MMF led to further increases in HO-1 
Fig. 6 Combination antiretroviral drug treatments induce the endogenous antioxidant response in primary neuroglial cultures. a, b Cortical neuroglial cultures were exposed to the indicated treatments (AZT, $25 \mu \mathrm{m}$; Rit, $10 \mu \mathrm{m}$; Saq, $1 \mu \mathrm{m}$ ) for $6 \mathrm{~h}$ to determine changes in NQO-1 (a) and HO-1 (b) mRNA levels. A representative of three experiments is shown. Actin was used as internal control and fold changes were determined by the $\Delta \Delta \mathrm{C}_{\mathrm{T}}$ method $\left({ }^{*} p<0.0001\right.$, oneway ANOVA, post-hoc

Newman-Keuls). c, d Whole cell lysates from cortical neuroglial cultures treated with the indicated drug combinations (AZT, $25 \mu \mathrm{m}$; Rit, $10 \mu \mathrm{m}$; Saq, $1 \mu \mathrm{m}$ ) for 16 and $48 \mathrm{~h}$ were immunoblotted for NQO-1 (c) and HO-1 (d).

Representative blots from three independent experiments are shown. Coomassie staining of gels were used as loading controls and fold changes over untreated lysates are indicated below each band of interest. e, f Whole cell lysates prepared from

hippocampus of rats treated for

7 days with AZT/Rit/Saq ( $n=4)$ or vehicle $(n=2)$ were

immunoblotted for $\mathrm{HO}-1$ and NQO-1 (e) and fold change in HO-1 protein levels in the cART group compared with the vehicle group is shown $(\mathbf{f})\left({ }^{*} p<0.05\right.$, Student's $t$ test)
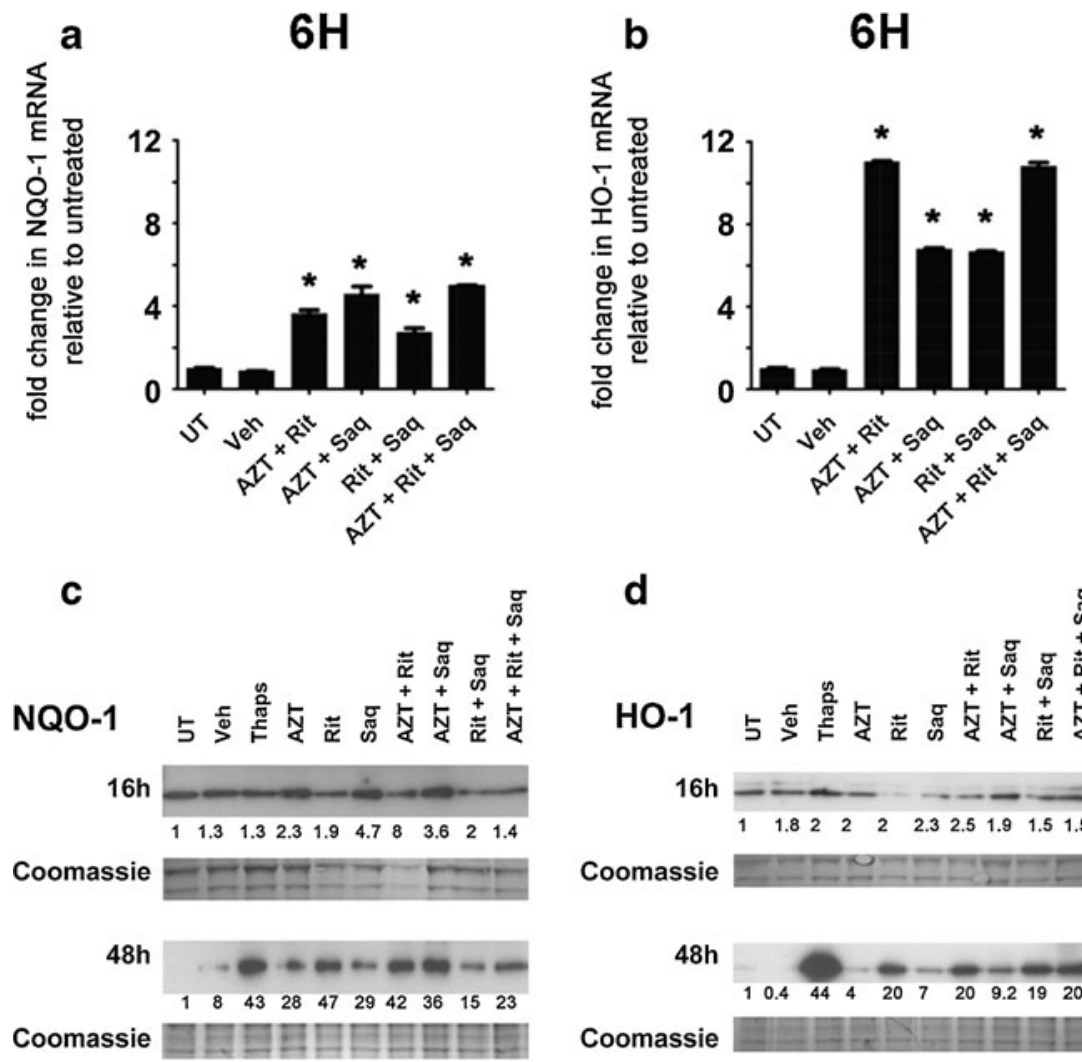

d
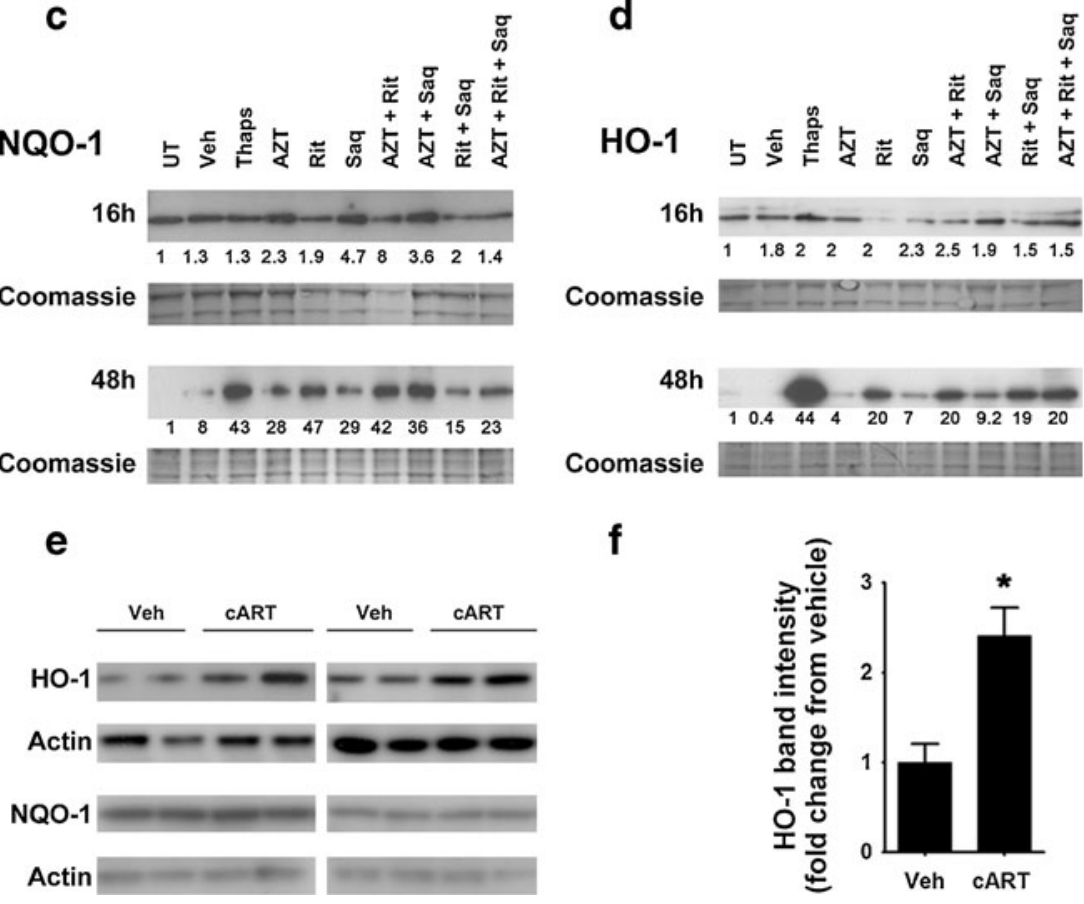

protein levels in ARV-treated cultures and provided protection against ARV-induced toxicity. Finally, our finding that the protection provided by MMF is reversed with chemical inhibition of HO-1 further provides evidence that ARV toxicity is mediated via induction of oxidative stress. The neuronal damage and death that occurs despite the cellular initiation of the endogenous antioxidant response following exposure to ARVs suggests that this response may be insufficient or too delayed to protect cultures from ARV toxicity. In support of this explanation, we observed that MMF-mediated augmentation of antioxidant responses is strongly protective against the neurotoxic effects of ARVs. Further studies using this in vitro model are warranted to determine whether other clinically relevant ARV drug combinations induce neurotoxicity and, if so, to establish whether similar pathways are involved.
DMF, a psoriasis treatment used in Europe since 1994, is currently being tested as a disease-modifying agent for multiple sclerosis (MS) (Anonymous 2011; Krieger 2011; Linker et al. 2011). MMF is the active DMF metabolite in vivo. Our report is the first to show MMF as a neuroprotectant against ARV-induced damage. Data from studies in MS patients show that DMF/MMF has good tolerability, can cross the BBB efficiently, and has relatively few and minor side effects. Furthermore, we have recently shown that DMF suppresses HIV replication, induces the antioxidant response in macrophaghes, and blocks neurotoxin release from macrophages (Cross et al. 2011). Overall, our findings make this immunomodulatory and antioxidant agent a good potential adjunctive therapeutic for use in HAND.

One of the paradoxical outcomes of cART is the persistence of HAND, despite successful viral control. Moreover, 

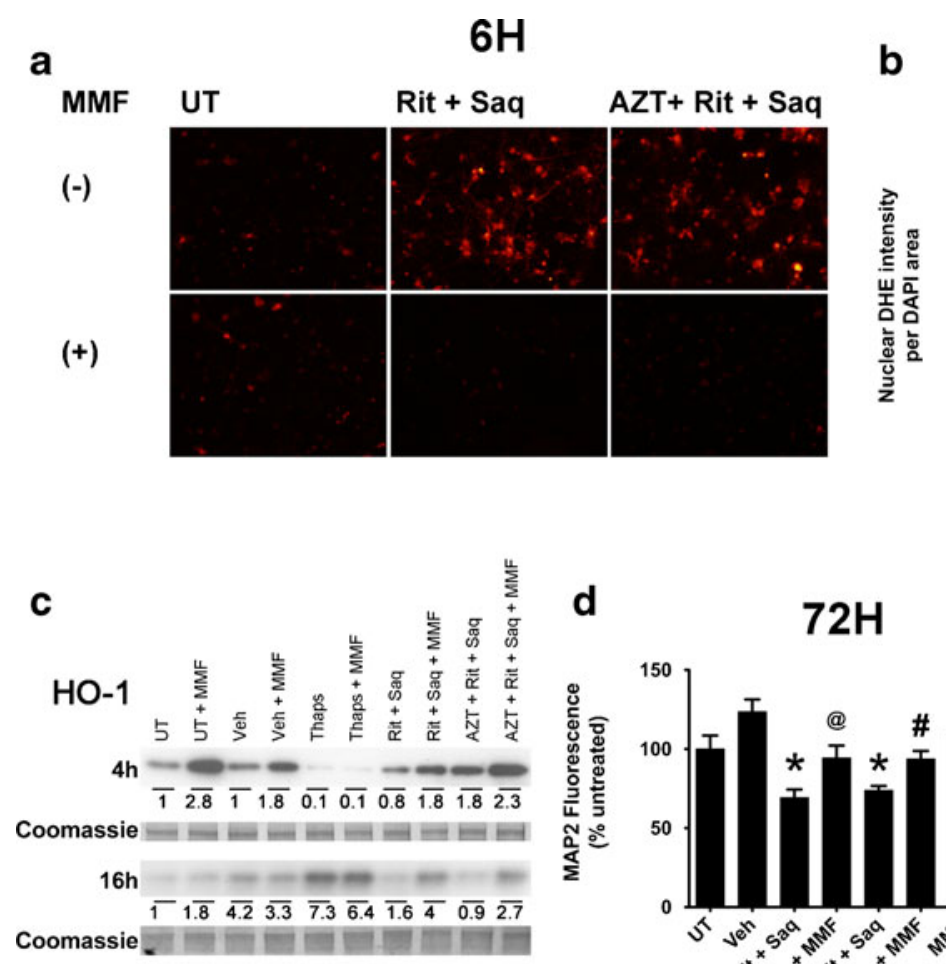
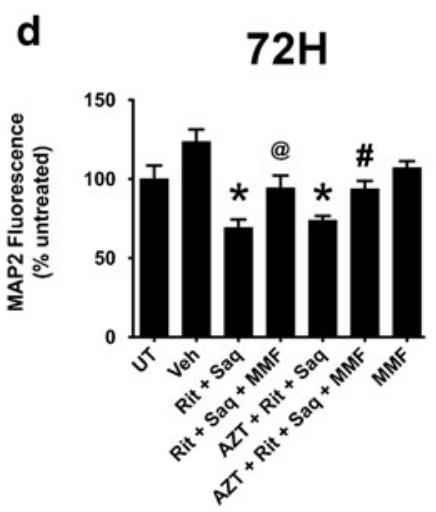

e

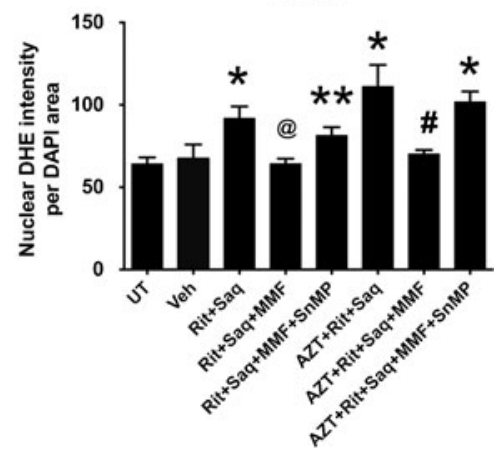

Fig. 7 MMF induces activation of a cellular antioxidant response and blocks neuronal damage/death. a In the absence (top) or presence (bottom) of MMF $(100 \mu \mathrm{M}), 21 \mathrm{DIV}$ rat neuroglial cultures on coverslips were treated for $6 \mathrm{~h}$ with Rit/Saq or AZT/Rit/Saq (AZT, $25 \mu \mathrm{m}$; Rit, $10 \mu \mathrm{m}$; Saq, $1 \mu \mathrm{m}$ ) or were left untreated. ROS generation was detected by DHE staining (red fluorescence). b Quantification of nuclear DHE was done as described above $\left(n=3 ;{ }^{*} p<0.01\right.$, one-way ANOVA with post-hoc Newman-Keuls). c Whole cell lysates of cultures exposed to Rit/Saq or AZT/ Rit/Saq treatments (AZT, $25 \mu \mathrm{m}$; Rit, $10 \mu \mathrm{m}$; Saq, $1 \mu \mathrm{m}$ ) in the absence or presence of MMF $(100 \mu \mathrm{M})$ for 4 or $16 \mathrm{~h}$ were immunoblotted for HO1. A representative blot from three independent experiments is shown. A coomassie band from the gel was used as loading control. Quantification of band intensities is shown under each corresponding lane. d Primary cortical neuroglial cultures were either pretreated with MMF $(100 \mu \mathrm{M})$ for 30 min or received no pretreatment; cultures were then treated with Rit/Saq or AZT/Rit/Saq (AZT, $25 \mu \mathrm{m}$; Rit, $10 \mu \mathrm{m}$; Saq, $1 \mu \mathrm{m}$ ) for $72 \mathrm{~h}$ to assess neuronal damage/death by MAP2 ELISA $(n=3$; vehicle $=0.04 \%$ DMSO; ${ }^{*} p<0.05$ vs. untreated; @ $p<0.05$ vs. Rit/Saq; $\# p<0.05$ vs. AZT/ Rit/Saq, one-way ANOVA with post-hoc Newman-Keuls). e Primary cortical neuroglial cultures were preincubated with SnMP $(20 \mu \mathrm{M})$ and/ or MMF $(100 \mu \mathrm{M})$ for $30 \mathrm{~min}$ before the addition of the indicated antiretroviral drugs (AZT, $25 \mu \mathrm{m}$; Rit, $10 \mu \mathrm{m}$; Saq, $1 \mu \mathrm{m}$ ) and were assessed for nuclear DHE accumulation at $16(n=3 ; * p<0.01$ vs. untreated; $* * p<0.05$ vs. untreated; @ $p<0.01$ vs. Rit/Saq; $\# p<0.05$ vs. AZT/Rit/ Saq, one-way ANOVA with post-hoc Newman-Keuls)

synaptic damage in the absence of neuronal loss, and these neuronal perturbations may contribute to, and may even precipitate, some of the clinical and pathological changes observed in the chronic course of HAND in the cART era. While detrimental, such slow damage also suggests that alterations in cART regimens to include ARVs with low neurotoxicity profiles, such as NRTIs or nNRTIs may halt synaptic damage, and provide a point where previous damage may be reversed, either due to the withdrawal of the toxic drug, or with the help of an adjuvant, such as fumaric acid esters. Future studies, first in primates, then in humans, will be crucial to explore the specific impact of treatment interruption on recovery from cART-mediated neuronal damage and to determine the eddicacy of potential adjunctive therapies necessary to mitigate the side effects of cART in the CNS. with a slower, nonetheless insidious changes including 
Acknowledgments We would like to thank Margaret Maronski for her help in the preparation of cortical cultures.

Grants This work was supported by the following National Institutes of Health Grants: MH083517 (K.J-S), NS043994 and NS27405 (D.L.K), AG000255 (S.A.C), DA22339 and DA18678 (R.C.P), CA133470 (M.C-S), and MH070306 (J.E.C.).

Conflict of interest All authors declare that they have no conflict of interest.

Open Access This article is distributed under the terms of the Creative Commons Attribution License which permits any use, distribution, and reproduction in any medium, provided the original author(s) and the source are credited.

\section{References}

Akay C, Lindl KA, Shyam N, Nabet B, Goenaga-Vazquez Y, Ruzbarsky J, Wang Y, Kolson DL, Jordan-Sciutto KL (2011a) Activation status of integrated stress response pathways in neurons and astrocytes of HAND cortex. Neuropathol Appl Neurobiol 118(6):1113-1123

Akay C, Lindl KA, Wang Y, White MG, Isaacman-Beck J, Kolson DL, Jordan-Sciutto KL (2011b) Site-specific hyperphosphorylation of $\mathrm{pRb}$ in HIV-induced neurotoxicity. Mol Cell Neurosci 47:154-165

Ances BM, Roc AC, Korczykowski M, Wolf RL, Kolson DL (2008) Combination antiretroviral therapy modulates the blood oxygen level-dependent amplitude in human immunodeficiency virusseropositive patients. J Neurovirol 14:418-424

Anderson PL, Rower JE (2010) Zidovudine and lamivudine for HIV infection. Clin Med Rev Ther 2:a2004

Anonymous (2011) Trial watch: phase III success for Biogen's oral multiple sclerosis therapy. Nature Reviews Drug Discovery 10:404

Anthonypillai C, Sanderson RN, Gibbs JE, Thomas SA (2004) The distribution of the HIV protease inhibitor, ritonavir, to the brain, cerebrospinal fluid, and choroid plexuses of the guinea pig. J Pharmacol Exp Ther 308:912-920

Anthonypillai C, Gibbs J, Thomas S (2006) The distribution of the antiHIV drug, tenofovir (PMPA), into the brain, CSF and choroid plexuses. Cerebrospinal Fluid Res 3:1

Bazzoli C, Jullien V, Le Tiec C, Rey E, Mentré F, Taburet A-M (2010) Intracellular pharmacokinetics of antiretroviral drugs in HIV-infected patients, and their correlation with drug action. Clin Pharmacokinet 49:17-45. doi:10.2165/11318110-000000000-000000000

Busidan Y, Dow-Edwards DL (1999) Neurobehavioral effects of perinatal AZT exposure in Sprague-Dawley adult rats. Neurotoxicol Teratol 21:359-363

Chen C, Kong AN (2004) Dietary chemopreventive compounds and ARE/EpRE signaling. Free Radic Biol Med 36:1505-1516

Cross SA, Cook DR, Chi AW, Vance PJ, Kolson LL, Wong BJ, JordanSciutto KL, Kolson DL (2011) Dimethyl fumarate, an immune modulator and inducer of the antioxidant response, suppresses HIV replication and macrophage-mediated neurotoxicity: a novel candidate for HIV neuroprotection. J Immunol 187:5015-5025

Cui L, Locatelli L, Xie MY, Sommadossi JP (1997) Effect of nucleoside analogs on neurite regeneration and mitochondrial DNA synthesis in PC-12 cells. J Pharmacol Exp Ther 280:1228-1234

Dore GJ, Correll PK, Li Y, Kaldor JM, Cooper DA, Brew BJ (1999) Changes to AIDS dementia complex in the era of highly active antiretroviral therapy. AIDS (London, England) 13:1249-1253

du Plooy M, Viljoen M, Rheeders M (2011) Evidence for time-dependent interactions between ritonavir and lopinavir/ritonavir plasma levels following P-glycoprotein inhibition in Sprague-Dawley rats. Biol Pharm Bull 34:66-70

Edén A, Fuchs D, Hagberg L, Nilsson S, Spudich S, Svennerholm B, Price RW, Gisslén M (2010) HIV-1 viral escape in cerebrospinal fluid of subjects on suppressive antiretroviral treatment. J Infect Dis 202:1819-1825

Fontes TM, Nakamura MU, Mattar R, Simoes RS, Wagner A, de Carvalho AM, Espiridiao S, Kulay L Jr (2011) Effects of the association zidovudine plus ritonavir on the liver and kidneys of pregnant rats. Morphological and biochemical aspects. Clin Exp Obstet Gynecol 38:126-130

Gannon P, Khan MZ, Kolson DL (2011) Current understanding of HIVassociated neurocognitive disorders pathogenesis. Curr Opin Neurol 24:275-283

Garvey L, Winston A, Walsh J, Post F, Porter K, Gazzard B, Fisher M, Leen C, Pillay D, Hill T, Johnson M, Gilson R, Anderson J, Easterbrook P, Bansi L, Orkin C, Ainsworth J, Palfreeman A, Gompels M, Phillips AN, Sabin CA (2011) Antiretroviral therapy CNS penetration and HIV-1-associated CNS disease. Neurology 76:693-700

Gupta RG, Kelly KM, Helke KL, Queen SE, Karper JM, Dorsey JL, Brice AK, Adams RJ, Tarwater PM, Kolson DL, Mankowski JL (2010) HIV and SIV induce alterations in CNS CaMKII expression and activation: a potential mechanism for cognitive impairment. Am J Pathol 176:2776-2784

Hazuda DJ, Young SD, Guare JP, Anthony NJ, Gomez RP, Wai JS, Vacca JP, Handt L, Motzel SL, Klein HJ, Dornadula G, Danovich RM, Witmer MV, Wilson KA, Tussey L, Schleif WA, Gabryelski LS, Jin L, Miller MD, Casimiro DR, Emini EA, Shiver JW (2004) Integrase inhibitors and cellular immunity suppress retroviral replication in rhesus macaques. Science 305:528-532

Heaton RK, Clifford DB, Franklin DR Jr, Woods SP, Ake C, Vaida F, Ellis RJ, Letendre SL, Marcotte TD, Atkinson JH, Rivera-Mindt M, Vigil OR, Taylor MJ, Collier AC, Marra CM, Gelman BB, McArthur JC, Morgello S, Simpson DM, McCutchan JA, Abramson I, Gamst A, Fennema-Notestine C, Jernigan TL, Wong J, Grant I (2010) HIVassociated neurocognitive disorders persist in the era of potent antiretroviral therapy: CHARTER Study. Neurology 75:2087-2096

Huisman MT, Smit JW, Wiltshire HR, Beijnen JH, Schinkel AH (2003) Assessing safety and efficacy of directed P-glycoprotein inhibition to improve the pharmacokinetic properties of saquinavir coadministered with ritonavir. J Pharmacol Exp Ther 304:596-602

Kageyama M, Namiki H, Fukushima H, Terasaka S, Togawa T, Tanaka A, Ito Y, Shibata N, Takada K (2005) Effect of chronic administration of ritonavir on function of cytochrome P450 3A and Pglycoprotein in rats. Biol Pharm Bull 28:130-137

Krieger S (2011) Multiple sclerosis therapeutic pipeline: opportunities and challenges. Mt Sinai J Med 78:192-206

Letendre S, Marquie-Beck J, Capparelli E, Best B, Clifford D, Collier AC, Gelman BB, McArthur JC, McCutchan JA, Morgello S, Simpson D, Grant I, Ellis RJ (2008) Validation of the CNS Penetration-Effectiveness rank for quantifying antiretroviral penetration into the central nervous system. Arch Neurol 65:65-70

Linker RA, Lee DH, Ryan S, van Dam AM, Conrad R, Bista P, Zeng W, Hronowsky X, Buko A, Chollate S, Ellrichmann G, Bruck W, Dawson K, Goelz S, Wiese S, Scannevin RH, Lukashev M, Gold R (2011) Fumaric acid esters exert neuroprotective effects in neuroinflammation via activation of the Nrf2 antioxidant pathway. Brain 134:678-692

Lledo-Garcia R, Nacher A, Casabo VG, Merino-Sanjuan M (2011) A pharmacokinetic model for evaluating the impact of hepatic and intestinal first-pass loss of saquinavir in the rat. Drug Metab Dispos 39:294-301

Mak IT, Kramer JH, Chen X, Chmielinska JJ, Spurney CF, Weglicki WB (2013) Mg-supplementation attenuates ritonavir-induced hyperlipidemia, oxidative stress and cardiac dysfunction in rats. Am J Physiol Regul Integr Comp Physiol 305(10):R1102-R1111. doi: 10.1152/ajpregu.00268.2013 
Manda VK, Mittapalli RK, Bohn KA, Adkins CE, Lockman PR (2010) Nicotine and cotinine increases the brain penetration of saquinavir in rat. J Neurochem 115:1495-1507

Marra CM, Zhao Y, Clifford DB, Letendre S, Evans S, Henry K, Ellis RJ, Rodriguez B, Coombs RW, Schifitto G, McArthur JC, Robertson K (2009) Impact of combination antiretroviral therapy on cerebrospinal fluid HIV RNA and neurocognitive performance. AIDS (London, England) 23:1359-1366

Mielke MM, Bandaru VV, McArthur JC, Chu M, Haughey NJ (2010) Disturbance in cerebral spinal fluid sphingolipid content is associated with memory impairment in subjects infected with the human immunodeficiency virus. J Neurovirol 16:445-456

Ngondi JL, Oben J, Forkah DM, Etame LH, Mbanya D (2006) The effect of different combination therapies on oxidative stress markers in HIV infected patients in Cameroon. AIDS Res Ther 3:19

Nolan D, Mallal S (2004) Complications associated with NRTI therapy: update on clinical features and possible pathogenic mechanisms. Antivir Ther 9:849-863

O'Donnell LA, Agrawal A, Jordan-Sciutto KL, Dichter MA, Lynch DR, Kolson DL (2006) Human immunodeficiency virus (HIV)-induced neurotoxicity: roles for the NMDA receptor subtypes. J Neurosci 26: 981-990

Pistell PJ, Gupta S, Knight AG, Domingue M, Uranga RM, Ingram DK, Kheterpal I, Ruiz C, Keller JN, Bruce-Keller AJ (2010) Metabolic and neurologic consequences of chronic lopinavir/ritonavir administration to C57BL/6 mice. Antiviral Res 88:334-342

Power C, Boisse L, Rourke S, Gill MJ (2009) NeuroAIDS: an evolving epidemic. Can J Neurol Sci 36:285-295

Reyskens KM, Essop MF (2013a) HIV protease inhibitors and onset of cardiovascular diseases: a central role for oxidative stress and dysregulation of the ubiquitin-proteasome system. Biochim Biophys Acta 6:691-700

Reyskens KM, Essop MF (2013b) The maladaptive effects of HIV protease inhibitors (lopinavir/ritonavir) on the rat heart. Int $\mathrm{J}$ Cardiol 168:3047-3049

Reyskens KM, Fisher TL, Schisler JC, O'Connor WG, Rogers AB, Willis MS, Planesse C, Boyer F, Rondeau P, Bourdon E, Essop MF (2013) Cardio-metabolic effectsof HIV protease inhibitors (lopinavir/ritonavir). PLoS One 8:e73347

Robertson K, Liner J, Meeker RB (2012) Antiretroviral neurotoxicity. J Neurovirol 18:388-399

Rodriguez-Pallares J, Parga J, Joglar B, Guerra M, Labandeira-Garcia J (2009) The mitochondrial atp-sensitive potassium channel blocker 5-hydroxydecanoate inhibits toxicity of 6-hydroxydopamine on dopaminergic neurons. Neurotox Res 15:82-95

Shibata N, Gao W, Okamoto H, Kishida T, Yoshikawa Y, Takada K (2002) In-vitro and in-vivo pharmacokinetic interactions of amprenavir, an HIV protease inhibitor, with other current HIV protease inhibitors in rats. J Pharm Pharmacol 54:221-229

Smurzynski M, Wu K, Letendre S, Robertson K, Bosch RJ, Clifford DB, Evans S, Collier AC, Taylor M, Ellis R (2011) Effects of central nervous system antiretroviral penetration on cognitive functioning in the ALLRT cohort. AIDS (London, England) 25:357-365, 310.1097/QAD.1090b1013e32834171f32834178

Thrivikraman KV, Huot RL, Plotsky PM (2002) Jugular vein catheterization for repeated blood sampling in the unrestrained conscious rat. Brain Res Brain Res Protocol 10:84-94

Touzet O, Philips A (2010) Resveratrol protects against protease inhibitorinduced reactive oxygen species production, reticulum stress and lipid raft perturbation. AIDS (London, England) 24:1437-1447

Tozzi V, Balestra P, Salvatori MF, Vlassi C, Liuzzi G, Giancola ML, Giulianelli M, Narciso P, Antinori A (2009) Changes in cognition during antiretroviral therapy: comparison of 2 different ranking systems to measure antiretroviral drug efficacy on HIV-associated neurocognitive disorders. JAIDS J Acquir Immune Defic Syndr 52: 56-63. doi:10.1097/QAI.1090b1013e3181af1083d1096
Tsai CC, Follis KE, Beck TW, Sabo A, Bischofberger N, Dailey PJ (1997) Effects of (R)-9-(2-phosphonylmethoxypropyl)adenine monotherapy on chronic SIV infection in macaques. AIDS Res Hum Retrovir 13:707-712

Vidal F, Gutierrez F, Gutierrez M, Olona M, Sanchez V, Mateo G, Peraire J, Vilades C, Veloso S, Lopez-Dupla M, Domingo P (2010) Pharmacogenetics of adverse effects due to antiretroviral drugs. AIDS Rev 12:15-30

Wagner A, Nakamura MU, Simoes RS, Oliveira-Filho RM, Fontes TM, de Carvalho LP, Espiridiao S, Kulay L Jr (2011) Chronic action of association of zidovudine, lamivudine and ritonavir on pregnant rats. A biologic assay. Clin Exp Obstet Gynecol 38:28-32

Wang Y, White MG, Akay C, Chodroff RA, Robinson J, Lindl KA, Dichter MA, Qian Y, Mao Z, Kolson DL, Jordan-Sciutto KL (2007) Activation of cyclin-dependent kinase 5 by calpains contributes to human immunodeficiency virus-induced neurotoxicity. J Neurochem 103:439-455

Wang Y, Shyam N, Ting JH, Akay C, Lindl KA, Jordan-Sciutto KL (2010) E2F1 localizes predominantly to neuronal cytoplasm and fails to induce expression of its transcriptional targets in human immunodeficiency virus-induced neuronal damage. Neurosci Lett 479:97-101

Waring JF, Ciurlionis R, Marsh K, Klein LL, Degoey DA, Randolph JT, Spear B, Kempf DJ (2010) Identification of proteasome gene regulation in a rat model for HIV protease inhibitor-induced hyperlipidemia. Arch Toxicol 84:263-270

Werth JL, Zhou B, Nutter LM, Thayer SA (1994) 2',3'-Dideoxycytidine alters calcium buffering in cultured dorsal root ganglion neurons. Mol Pharmacol 45:1119-1124

White MG, Wang Y, Akay C, Lindl KA, Kolson DL, Jordan-Sciutto KL (2011) Parallel high throughput neuronal toxicity assays demonstrate uncoupling between loss of mitochondrial membrane potential and neuronal damage in a model of HIV-induced neurodegeneration. Neurosci Res 70:220-229

Wilcox KS, Buchhalter J, Dichter MA (1994) Properties of inhibitory and excitatory synapses between hippocampal neurons in very low density cultures. Synapse 18:128-151

Wynn HE, Brundage RC, Fletcher CV (2002) Clinical implications of CNS penetration of antiretroviral drugs. CNS Drugs 16:595-609

Xu J, Ikezu T (2009) The comorbidity of HIV-associated neurocognitive disorders and Alzheimer's disease: a foreseeable medical challenge in post-HAART era. J NeuroImmune Pharm 4:200-212

Yang Y, Dahly-Vernon AJ, Blomme EA, Lai-Zhang J, Kempf DJ, Marsh KC, Harrington YA, Nye SH, Evans DL, Roman RJ, Jacob HJ, Waring JF (2010) Liver transcriptomic changes associated with ritonavir-induced hyperlipidemia in sensitive and resistant strains of rats. Vet J 185:75-82

Yilmaz A, Ståhle L, Hagberg L, Svennerholm B, Fuchs D, Gisslén M (2004) Cerebrospinal fluid and plasma HIV-1 RNA levels and lopinavir concentrations following lopinavir/ritonavir regimen. Scand J Infect Dis 36:823-828

Yilmaz A, Gisslen M, Spudich S, Lee E, Jayewardene A, Aweeka F, Price RW (2009) Raltegravir cerebrospinal fluid concentrations in HIV-1 infection. PLoS One 4:e6877

Zhao S, Zhang Y, Gu Y, Lewis DF, Wang Y (2004) Heme oxygenase-1 mediates up-regulation of adhesion molecule expression induced by peroxynitrite in endothelial cells. J Soc Gynecol Investig 11:465-471

Zink MC, Suryanarayana K, Mankowski JL, Shen A, Piatak M Jr, Spelman JP, Carter DL, Adams RJ, Lifson JD, Clements JE (1999) High viral load in the cerebrospinal fluid and brain correlates with severity of simian immunodeficiency virus encephalitis. J Virol 73:10480-10488

Zink MC, Brice AK, Kelly KM, Queen SE, Gama L, Li M, Adams RJ, Bartizal C, Varrone J, Rabi SA, Graham DR, Tarwater PM, Mankowski JL, Clements JE (2010) Simian immunodeficiency virus-infected macaques treated with highly active antiretroviral therapy have reduced central nervous system viral replication and inflammation but persistence of viral DNA. J Infect Dis 202:161-170 